\title{
Efficient Power-Aware Dynamic Source Routing Protocol
}

\author{
by \\ YANJUAN CHENG

\begin{abstract}
A thesis
submitted to the School of Mathematics and Statistics

and the Faculty of Graduate Studies and Research

in partial fulfillment of the requirements

for the degree of

Master of Science, Information and Systems Science
\end{abstract}

School of Mathematics and Statistics

Ottawa-Carleton Institute of Mathematics and Statistics

Carleton University

Ottawa, Ontario

Canada

March 16, 2004

(C) Copyright 2004, Yanjuan Cheng 


\author{
National Library \\ of Canada \\ Acquisitions and \\ Bibliographic Services \\ 395 Wellington Street \\ Ottawa ON K1A ON4 \\ Canada
}

Bibliothèque nationale

du Canada

Acquisisitons et services bibliographiques

395 , rue Wellington Ottawa ON K1A ON4 Canada
Your file Votre référence ISBN: 0-612-93976-6 Ourfile Notre référence ISBN: 0-612-93976-6
The author has granted a nonexclusive licence allowing the National Library of Canada to reproduce, loan, distribute or sell copies of this thesis in microform, paper or electronic formats.

The author retains ownership of the copyright in this thesis. Neither the thesis nor substantial extracts from it may be printed or otherwise reproduced without the author's permission.
L'auteur a accordé une licence non exclusive permettant à la Bibliothèque nationale du Canada de reproduire, prêter, distribuer ou vendre des copies de cette thèse sous la forme de microfiche/film, de reproduction sur papier ou sur format électronique.

L'auteur conserve la propriété du droit d'auteur qui protège cette thèse. $\mathrm{Ni}$ la thèse ni des extraits substantiels de celle-ci ne doivent être imprimés ou aturement reproduits sans son autorisation.
In compliance with the Canadian Privacy Act some supporting forms may have been removed from this dissertation.

While these forms may be included in the document page count, their removal does not represent any loss of content from the dissertation.
Conformément à la loi canadienne sur la protection de la vie privée, quelques formulaires secondaires ont été enlevés de ce manuscrit.

Bien que ces formulaires aient inclus dans la pagination, il n'y aura aucun contenu manquant. 
PAGINATION ERROR.

TEXT COMPLETE.
ERREUR DE PAGINATION.

LE TEXTE EST COMPLET. 


\begin{abstract}
Wireless networking has witnessed an explosion of interest from consumers in recent years for its application in mobile communication. The mobile wireless networks are power constrained because of the nodes' limited battery energy. In order to maximize the lifetime of networks, energy consumption of each node should be controlled through network-related transactions such that the energy dissipation rate of each node could be balanced among the whole network.

This thesis proposes a protocol, namely EPDSR (efficient power-aware dynamic source routing protocol), based on DSR (dynamic source routing protocol). New routing algorithms are added in route discovery. These include a power-aware source routing algorithm and a two-path discovery algorithm. Compared with the DSR, the route maintenance algorithm in EPDSR is improved by considering energy depletion of the nodes as well as mobility. Simulation in this thesis focuses on sparse networks since lifetime of sparse networks reduces quickly due to the overuse of small sets of nodes. Simulation results demonstrate that the protocol EPDSR can significantly increase the lifetime of networks and extend the connection of the network nodes. The number of dead nodes in EPDSR decreases around 22\% compared with DSR. The protocol EPDSR greatly reduces energy variance by about $37 \%$. It prevents overuse of a small set of nodes, and ultimately extends the network partition time. However, average energy consumption for packet transmission per byte in EPDSR increases approximately by $14 \%$.
\end{abstract}




\section{Acknowledgements}

I would like to take this opportunity to express my gratitude to my thesis supervisor, Dr. Evangelos Kranakis, for his technical guidance, enthusiastic support and discussion. His earnest research attitude often inspires me with valuable ideas during my thesis research.

I would like to thank the professors at the School of Mathematics and Statistics, School of Computer Science and Department of Systems and Computer Engineering, Carleton University for all the things I learned here.

I would like to acknowledge the suggestions, encouragement and help from Mr. Jinliang Liu, Ms. Jianzhong Li, Ms. Yanru Zhang, Ms. Xuemei Liang, Ms. Liping Zhao, Ms. Chaohui Zhang, Ms. Weina Yuan, Ms. Xia Xu, and Ms. Yingjun Zhu. I also want to thank them for bringing me so much joy.

Finally, I leave my special thanks to my dearest parents Mr. Weifang Cheng and Mrs. Shuqin Yu, my wonderful husband Jijun Liu, my brother Jingbo Cheng and sister-in-law Chunjie Wang for their understanding, support and encouragement. This thesis is dedicated to my family, especially to my lovely niece Yanhan. 


\section{List of contents}

ABSTRACT ........................................................................

ACKNOWLEDGEMENTS ............................................... IV

LIST OF CONTENTS ....................................................................... V

LIST OF FIGURE ...................................................................IX

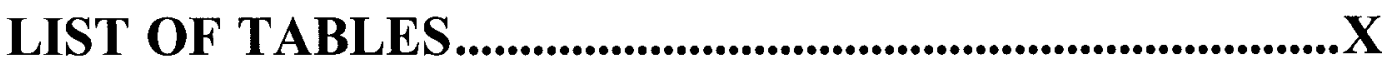

LIST OF ACRONYMS .................................................................. XI

CHAPTER 1 INTRODUCTION .........................................................1

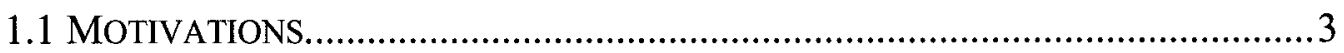

1.2 RESEARCH OVERVIEW AND CONTRIBUTIONS ...........................................4

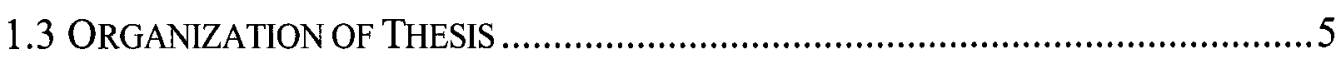

CHAPTER 2 MANET BACKGROUND......................................7

2.1 TABLE DRIVEN Routing Protocols ................................................. 10

2.1.1 Destination-Sequenced Distance-Vector Routing Protocol..................10

2.1.2 Cluster-Head Gateway Switch Routing .............................................11

2.1.3 Global State Routing.................................................................. 13

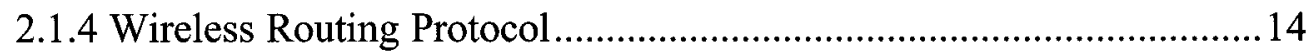

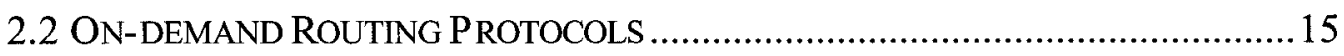

2.2.1 Temporally-Ordered Routing Algorithm......................................... 15

2.2.2 Ad-hoc On-Demand Distance Vector Routing............................... 18

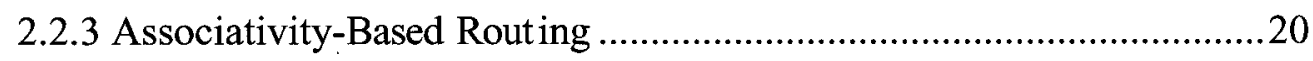

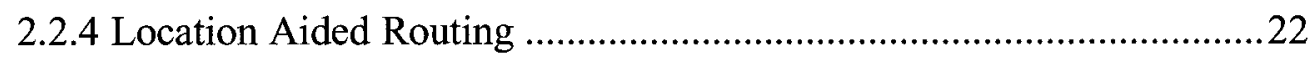




\section{CHAPTER 3 ENERGY-RELATED METRICS IN}

NETWORK PROTOCOL ................................ 24

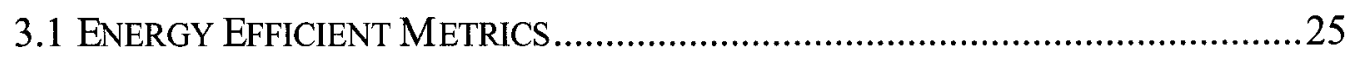

3.1.1 Maximize network lifetime (Time to network partition)....................26

3.1.2 Minimize power variance among mobile nodes ..............................27

3.1.3 Minimize energy consumption per packet.....................................27

3.1.4 Minimize maximum node cost .....................................................28

3.2 ClassifiCATION OF CONSERVING ENERgY Routing Protocols ................30

3.2.1 Switching on/off transmitters to save energy ......................................30

3.2.2 Topology control by adaptively adjusting radio power ........................32

3.2.3 Routing based on the metric of energy consumption ........................33

3.2.3.1 Minimum Total Transmission Power Routing ............................. 33

3.2.3.2 Minimum Battery Cost Routing ................................................ 35

3.2.3.3 Min-Max Battery Cost Routing ................................................. 37

3.2.3.4 Conditional Min-Max Battery Cost Routing ................................ 38

\section{CHAPTER 4 DSR PROTOCOL DESCRIPTION AND}

IMPLEMENTATION IN NS2 $\ldots . . . \ldots \ldots \ldots \ldots \ldots \ldots \ldots . . . . . . . . .40$

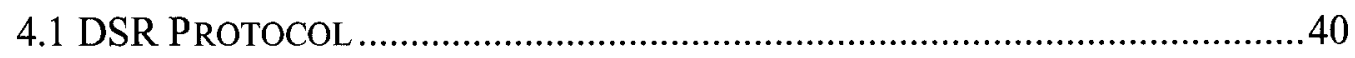

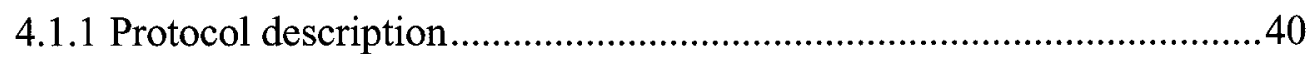

4.2 SIMULATION SETUP AND SYSTEM PARAMETERS.......................................45

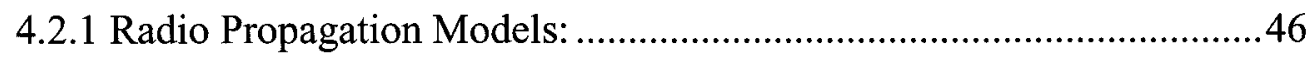

4.2.2 Media Access Control Protocols: ...................................................46

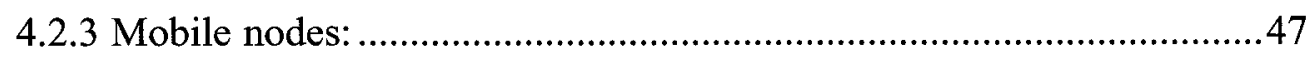

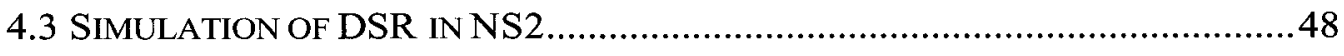

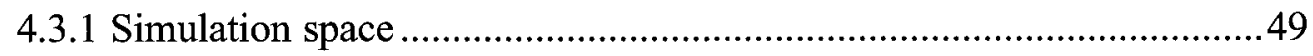

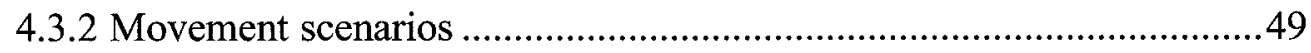

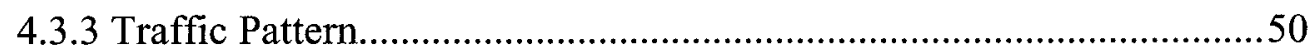


4.3.4 Simulation Time .51

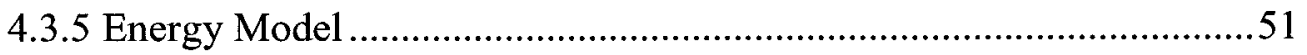

4.3.6 Performance metrics.......................................54

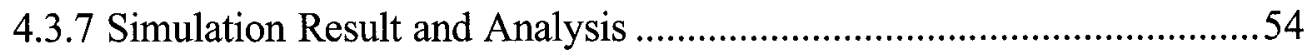

4.3.7.1 Network lifetime (Number of dead nodes) ..................................54

4.3.7.2 Difference of nodal energy .....................................................5 57

\section{CHAPTER 5 EFFICIENT POWER-AWARE DYNAMIC}

SOURCE ROUTING PROTOCOL SIMULATION.... 59

5.1 SIMULATION BASED ON DSR …………………......................................59

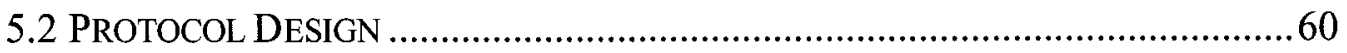

5.2.1 Multipath route discovery.............................................................60

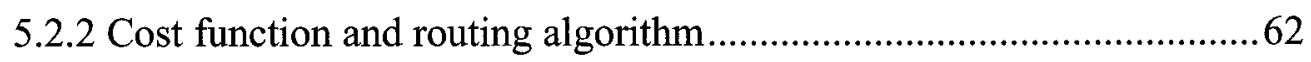

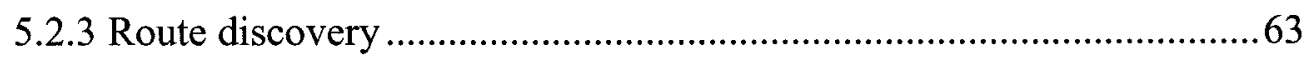

5.2.3.1 Intermediate nodes receive RREQ ............................................64

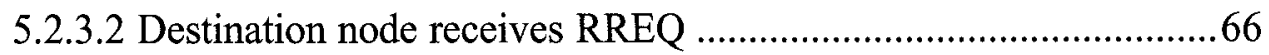

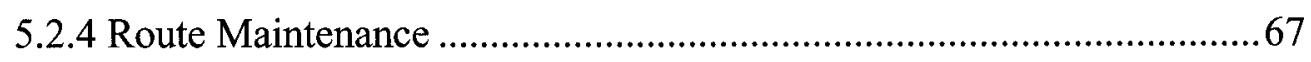

5.2.5 Difference between EPDSR and DSR ............................................69

5.2.6 Difference between DSR, MTPR, MBCR, and MMBCR ...................70

5.3 SIMULATION RESULT AND PERFORMANCE ANALYSIS ............................... 71

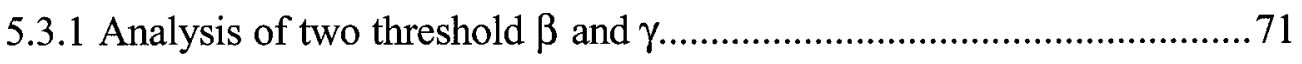

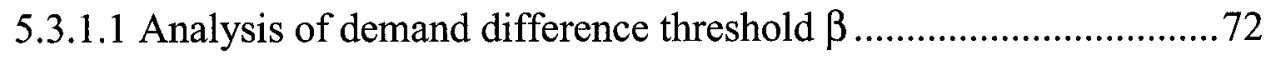

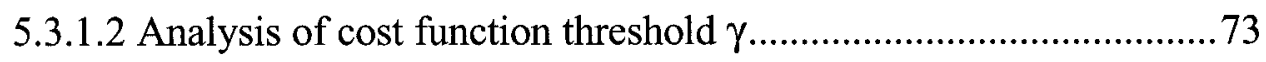

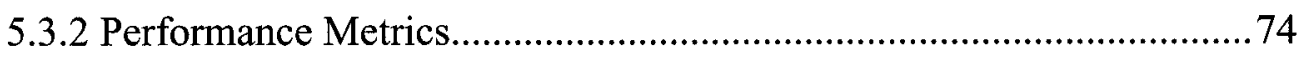

5.3.3 Performance Comparison when Varying Mobility..............................75

5.3.3.1 Network lifetime (dead node number)..........................................75

5.3.3.2 Nodal Energy difference ........................................................... 78

5.3.3.3 Energy consumption per byte packet...........................................79

5.3.3.4 Summary of EPDSR performance analysis .................................81 
5.3.4 Performance comparison when varying density..............................8 82

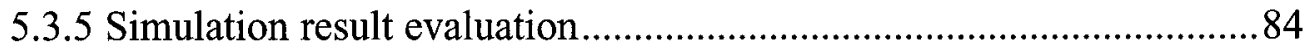

CHAPTER 6 CONCLUSIONS AND FUTURE WORK........... 87

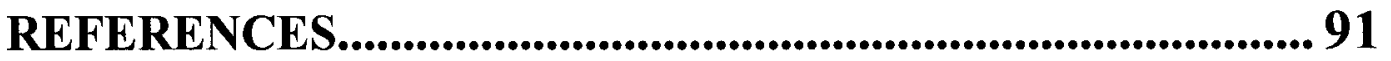

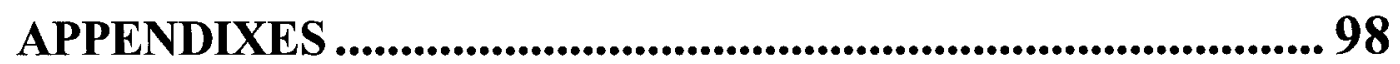

1. ENERGY EFFICIENT ROUTING PROTOCOL SIMULATION USING NS2 .................98

2. NS2 MOBILE NODE NETWORK COMPONENTS.............................................98

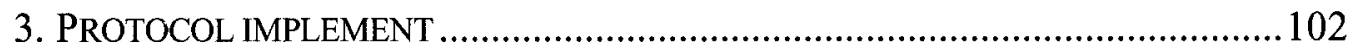

4. MobILE NODE MOVEMENT SCENARIO FILES CREATION ................................ 103

5. TRAFFIC PATTERN SCENARIo FILES CREATION .........................................103 


\section{List of Figure}

Figure 1. Infrastructured and infrastructureless network ..............................8

Figure 2. Categorization of ad hoc routing protocols......................................9

Figure 3. CGSR routing from node $S$ to node $D$.................................................12

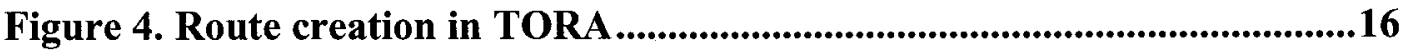

Figure 5. Route maintenance for source and destination movement in ABR..20

Figure 6. Mobile network communication ......................................................26

Figure 7. Three states in BECF ...........................................................................31

Figure 8. An illustration of route selection using different algorithms .............34

Figure 9. Route discovery in DSR: broadcasting RREQ ....................................42

Figure 10. Route discovery in DSR: propagation of the route reply ................43

Figure 11. Number of dead nodes as function of pause time ...............................55

Figure 12. Number of dead nodes as function of node speed..........................56

Figure 13. Difference of nodal energy with different scenarios..........................57

Figure 14. Flowchart when intermediate nodes receive RREQ ........................65

Figure 15. Route discovery number with different demand difference thresholds .............................................................................72

Figure 16. Route discovery number with different cost function thresholds ...73

Figure 17. Comparison of dead node number (node speed: $10 \mathrm{~m} / \mathrm{s}$ ).................76

Figure 18. Comparison of dead node number (node speed: $30 \mathrm{~m} / \mathrm{s}$ )..................77

Figure 19. Nodal energy difference in DSR and EPDSR ..................................79

Figure 20. Energy consumption per byte packet.................................................79

Figure 21. Mobile node network components in DSR...................................100 


\section{List of Tables}

Table 1. Simulation Parameters ......................................................................48

Table 2. Energy model parameters ........................................................................52

Table 3. Difference between EPDSR and DSR................................................69

Table 4. Difference between EPDSR and other power-aware protocols......70

Table 5. Average values of EPDSR performance with varying mobility ......81

Table 6. Average values of EPDSR performance with varying density ........83

Table 7. Simulation result evaluation in networks ........................................85 


\section{List of Acronyms}

ABR: Associativity Based Routing

ACK: Acknowledgment

AODV: Ad-hoc On-Demand Distance Vector Routing

ARP: Address Resolution Protocol

CBR: Constant Bit Rate

CGSR: Cluster-Head Gateway Switch Routing

CMMBCR: Conditional Min-Max Battery Cost Routing

DAG: Directed Acyclic Graph

DCM: Distributed Coordination Function

DSDV: Destination Sequenced Distance Vector Routing

DSR: Dynamic Source Routing

FORP: Flow Oriented Routing Protocol

GPS: Global Position System

GSR: Global State Routing

IMEP: Internet MANET Encapsulation Protocol

IP: Internet Protocol

LAR: Location Aided Routing

LCC: Least Cluster Change

LL: Link Layer

LMR: Lightweight Mobile Routing 
MAC: Media Access Control

MANET: Mobile Ad Hoc Network

MBCR: Minimum Battery Cost Routing

MMBCR: Min-Max Battery Cost Routing

MTPR: Minimum Total Transmission Power Routing

NPDU: Network Protocol Data Units

NAM: Network Animator

NS2: Network Simulator

QoS: Quality of service

RREQ: Route Request Packet

RREP: Route Reply Packet

RTS/CTS: Request To Send/Clear to Send

SSA: Signal Stability-based Adaptive Routing

TCP: Transmission Control Protocol

TORA: Temporally Ordered Routing Algorithm

WRP: Wireless Routing Protocol 


\section{Chapter 1}

\section{Introduction}

Mobile ad hoc network (MANET) [9] is an emerging new technology and it has been experiencing a tremendous rise in popularity.

A MANET is a collection of mobile wireless nodes that can dynamically form a network without necessarily using any fixed network infrastructure or central administration. Mobile networks allow users to access information and services electronically, regardless of their geographic position. MANET is a typical multi hop network. The communication between two nodes, which are not in the same transmission range, is completed by the intermediate nodes. In recent years, mobile computing has been becoming more and more popular. However, the mobile ad hoc topology may change when hosts move. Furthermore, some features of MANET make it a big challenge to develop a perfect mobile ad hoc routing protocol. These include 1) dynamic topology; 2) bandwidth-constrained; 3) energy-constrained, and 4) limited physical security. This thesis will focus on improving the protocol by finding some remedy to feature 3 ).

A MANET consists of wireless hosts that move around. All the hosts have no permanent physical location. In order to facilitate communication within the mobile network, a routing protocol is used to discover routes between nodes before the exchange of data packets. In recent years, more and more unicast routing protocols 
have been proposed and used to create and maintain the communication between mobile nodes. The MANET routing protocols in ad hoc wireless networks are generally categorized into two kinds: proactive (also called table-driven) and reactive (also called on-demand). In proactive protocols, nodes keep track of routes to all destinations. While in reactive protocols, nodes keep track of only those destinations of immediate interest.

In proactive protocols, each host maintains one or more tables to store up-to-date routing information and propagates updated information throughout the network even if it never uses that route to some destination. These protocols maintain valid routes to all communication mobile nodes all the time during the simulation. Periodic route updates are exchanged in order to synchronize the tables. The protocols propagate and maintain routing information, regardless of whether or not it is needed. This will result in extra overhead. The route maintenance in proactive protocols can waste the limited bandwidth and a lot of limited battery power. However, since the route is always available for any transmission, the transmission latency may be reduced. Some examples of table-driven ad hoc routing protocols include Destination Sequenced Distance Vector Routing (DSDV), Wireless Routing Protocol (WRP) and Cluster-head Gateway Switch Routing (CGSR).

In contrast, the reactive protocols create routes only when needed by a source node. Therefore a route discovery process is required within the network. Once a route has been established, it is maintained by a route maintenance procedure until 
either the destination becomes inaccessible or the route is not needed any more. Each node may use less bandwidth and battery power to maintain and update the route tables. But the latency will increase because the source waits to find route through route discovery procedure before transmitting messages. Typical examples of source initiated ad hoc routing protocols include Dynamic Source Routing (DSR) [15] and Ad Hoc On-Demand Distance Vector (AODV) [30].

\subsection{Motivations}

In ad hoc networks, mobile devices are battery-operated and the battery technology has not been improving rapidly. Therefore, power consumption is likely to remain an issue in mobile wireless network routing. Extending the network lifetime has become an important goal. There have been many proposals to incorporate power-awareness into MANET routing designs. Since communication of two nodes may relay on other intermediate nodes, the death of a small set of nodes with depleted power might cause partitioning of the entire network. Conventional routing protocols do not consider the power budget where the routes between nodes are built by the shortest path routing algorithm. When the same algorithm is used in MANET, it may lead to a quick depletion of the energy of a few nodes overused in the path. Several power-aware routing algorithms, such as Minimum Total transmission Power Routing (MTPR) [40], Minimum Battery Cost Routing (MBCR) [40], and Min-Max Battery Cost Routing (MMBCR) [40], have been presented. All of these algorithms concentrate on different power aspects and 
have their own advantages and disadvantages. This thesis will analyze these protocols in details such as route discovery, cost function setting, and the advantage and the disadvantage of each protocol. The thesis eventually proposes a new and more efficient protocol, energy efficient power-aware dynamic source routing (EPDSR), based on DSR.

\subsection{Research Overview and Contributions}

This thesis focuses on developing a new protocol that will increase the node lifetime and eventually extend the overall lifetime of the entire ad hoc network. This will be done by improving the power consumption balance among the nodes and the connection of the network.

In most existing protocols, a mobile node may consume all its energy to participate in the operation, without considering the remaining energy. Instead, in the energy efficient protocol EPDSR proposed in this thesis, each node will only use part of energy to transmit the data packets. This is done through a route discovery procedure. This new protocol uses a cost function to decide route selection, instead of using the traditional shortest hop algorithm.

The proposed routing protocol EPDSR in this thesis is based on the reactive protocol DSR. The reason that DSR is used as our base model is mainly due to the fact that it is a typical on-demand protocol with less bandwidth and energy use. Comparisons between EPDSR and DSR are made in three aspects: the number of 
dead nodes (network lifetime), energy variance among nodes, and energy consumption per byte packet. Results from this thesis clearly show that the proposed protocol EPDSR leads to fewer dead nodes, more effective power balance among nodes, and more energy consumption for data transmission.

In summary, the most significant contribution of this thesis is the proposed routing protocol, EPDSR. In particular, some of the major contributions include:

1. Proposed a new route discovery algorithm that considers the remaining energy for each node and uses a cost function to choose the best power-saving route.

2. Proposed a new route maintenance algorithm that deals with the broken routes due to the nodal energy depletion and node mobility.

\subsection{Organization of Thesis}

This thesis is structured into six chapters. Following this introductory chapter, existing wireless ad hoc network routing protocols will be reviewed in Chapter 2. Two categories of protocols are discussed, table-driven and on-demand; Chapter 3 introduces some power-aware routing protocols such as MTPR, MBCR, MMBCR, and CMMBCR. Some energy-related metrics are also presented. Chapter 4 briefly reviews dynamic routing protocol, introduces the network simulator (NS2), presents the simulation results in DSR with different mobility and pause time, and analyzes the simulation results from a set of performance metrics. Based on previous analyses, Chapter 5 proposes the protocol EPDSR with the energy efficient two-path and cost function route discovery and route maintenance algorithm; implements the 
algorithm based on DSR; compares EPDSR with the existing protocols such as DSR, MTPR, MBCR and MMBCR; compares the simulation result under different value of mobility rate and pause time; analyzes the simulation results from different perspectives such as network lifetime, nodal energy difference and energy consumption per byte packet. Conclusions and suggestions for future studies will be introduced in Chapter 6. 


\section{Chapter 2}

\section{MANET Background}

Wireless network is an emerging new information technology that will allow users to access information and services electronically, regardless of their geographic position In the past decades, wireless networks have become increasingly popular in communication and computing industry. This is due to recent technological advances in laptop computers and wireless data communication devices such as wireless modems and wireless LANs. There are currently two variations of mobile wireless networks: infrastructured network and infrastructureless network.

Infrastructured networks consist of a network with fixed and wired gateways and mobile nodes. A mobile host communicates with a bridge, called base station, within its communication range. Base stations are connected to the gateways between the base station and mobile node. A mobile node can move geographically while communicating. The mobile node, in the area of wireless transmission range covered by at least one base station, communicates with base stations by which exchanges information with other mobile nodes. When it moves out of the range of one base station, it connects with another new base station and starts communicating through it. Mobile communication in infrastructured networks is single-hop communication. 
In Figure 1(a), G is a fixed gateway, B is a base station, and nodes A, D, and C are mobile nodes. In this example, the transmission route from node A to D could be A-B1-G2-G1-G3-B2-D and the communication between nodes D and C could be through route D-B2-C.

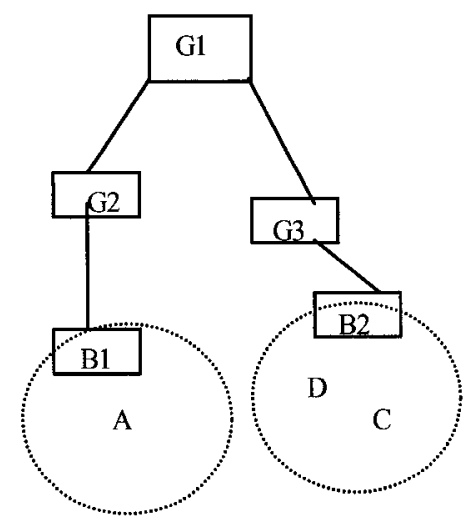

(a) Example of infrastructure

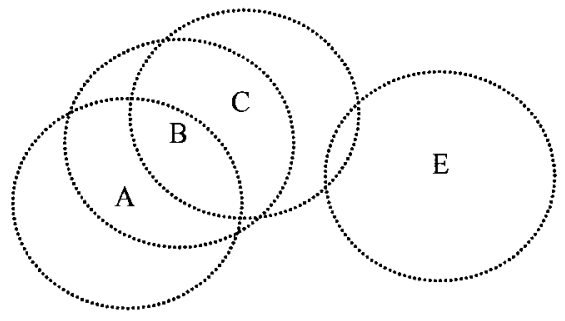

(b) Example of infrastructureless

Figure 1. Infrastructured and infrastructureless network

Infrastructureless mobile networks, such as mobile ad hoc networks, have no fixed routers. All nodes are capable of movement and can be connected dynamically in an arbitrary manner. All nodes of these networks behave as routers and take part in discovery and maintenance of routes to the other nodes in the network. Communication between mobile nodes in infrastructureless network is multi-hop communication. In Figure 1(b), the circle is the transmission range of mobile nodes inside it and the node is at the centre of transmission range. The route between node A and C could be A-B-C, and there is no route between A and E in this topology. Ad hoc networks are very useful in emergency search-and-rescue operations, 
meetings or conventions in which persons wish to quickly share information and data acquisition operations in inhospitable terrain.

MANET is an autonomous system composed of a group of mobile nodes that are dynamically and arbitrarily linked without central administration. Nodes form a network independently of any fixed station infrastructure. MANET is generally characterized by bandwidth-constrained, variable-capacity links and unpredictable topology. MANET is a typical multi-hop network with several characteristics: 1) Mobile network topology structure change is unpredictably; 2) Nodes are batterypowered and energy-constrained and battery lifetime of nodes is limited. Due to unpredictability and battery-constraint in MANET, designing an efficient ad hoc network protocol poses a significant technical challenge.

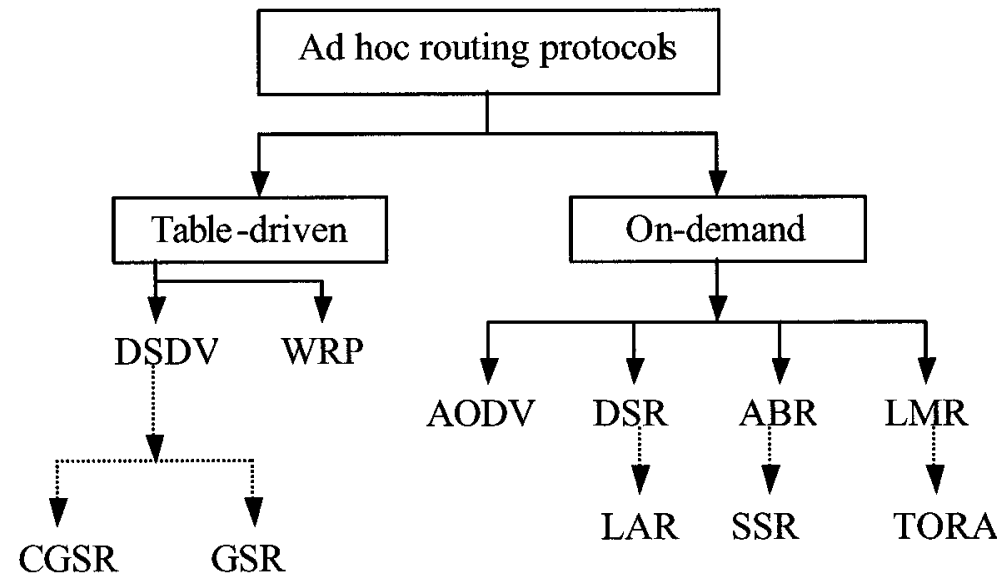

Figure 2. Categorization of ad hoc routing protocols

Many ad hoc routing protocols have been proposed and are generally categorized in two groups: proactive (table-driven) and reactive (on-demand). The difference between two kinds of protocols is based on when and how to discover 
and update the route. In proactive protocols, each node maintains a consistent route information table, whereas in reactive routing protocols, routes are discovered only when desired by the source node.

Solid lines in Figure 2 [36] represent direct descendants, while dotted lines depict logical descendants. Despite being designed for the same type of underlying network, the characteristics of each protocol are quite distinct. This section describes protocols and categorizes them according to their characteristics.

\subsection{Table Driven Routing Protocols}

Table-driven routing protocols attempt to maintain consistent and up-to-date routing information from each node to every other node in the network. These protocols require each node to maintain one or more tables to store routing information, and they respond to changes in network topology by propagating updates throughout the network in order to maintain a consistent network view. Routing protocols differ in the distribution of changed information in the network and the number of necessary routing-related tables.

\subsubsection{Destination-Sequenced Distance-Vector Routing Protocol}

Destination-Sequenced Distance-Vector (DSDV) [31] Routing Algorithm is based on the classical Bellman-Ford Routing Algorithm with certain improvement. Every mobile station in the network maintains and updates a routing table recording all possible destinations, the number of hops to reach the destination and the sequence number assigned by the destination node. Sequence numbers enable 
mobile nodes to distinguish stale routes from new ones, thereby avoiding the formation of routing loops. DSDV uses both periodic and triggered routing updates to maintain table consistency. Triggered routing updates are used when network topology structure changes are detected in order to propagate routing information immediately. Table information update is both time-driven and event-driven. Stations use two methods to deliver the routing table information: a full dump or an incremental update. In a full dump, the station sends all the available routing information to the neighbours. In an incremental update, only entries from the routing table that has a metric change since the last update are sent. If there is space in the incremental update packet, those entries whose sequence number has been changed may be included. When the network is relatively stable, incremental updates are sent to avoid extra traffic and full dump is relatively infrequent. Otherwise, in a fast-changing network, incremental packets can grow big so full dump will be more frequent. Each route update packet, in addition to routing table information, also carries a unique sequence number assigned by the transmitter. The route labelled with the highest sequence number is used. If two routes have the same sequence number, the route with the best metric (i.e. shortest route) is used.

\subsubsection{Cluster-Head Gateway Switch Routing}

Cluster-Head Gateway Switch Routing (CGSR) [8] is based on DSDV Routing algorithm with some improvement.

The mobile nodes are aggregated into clusters controlled by the selected clusterheads. All mobile nodes in the communication range of the cluster-head belong to 
its cluster. A gateway node is a node that is in the communication range of two or more cluster-heads. In a dynamic network, the disadvantage of cluster head scheme is that frequent cluster head changes can cause routing protocol performance degradation. Then CGSR uses Least Cluster Change (LCC) algorithm to prevent frequent cluster head changes. In LCC algorithm, only two conditions could cause cluster head changes: 1) Two cluster-heads come into one cluster; 2) One of the nodes moves out of the range of all the cluster-heads.

The LCC algorithm works as follows. When the source generates packet, it sends this packet to its cluster-head. Through this cluster-head, the packet is transmitted to the gateway node connecting cluster-heads along the route to the destination. The gateway sends it to that cluster-head and this step continues until the destination cluster-head is reached. The destination cluster-head forwards the

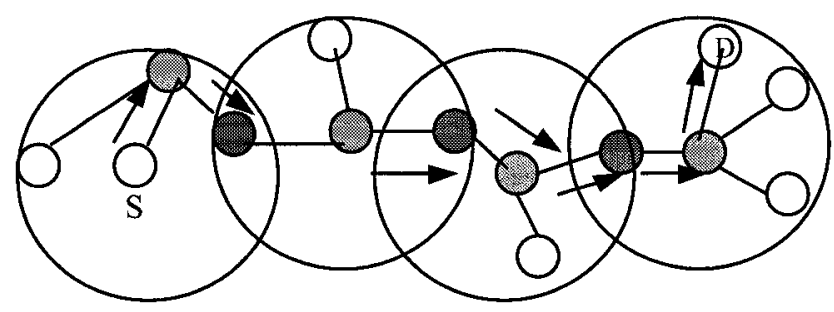

Internal node

Cluster head

Gateway node

Figure 3. CGSR routing from node $S$ to node $D$

data packet to the destination.

Figure 3 shows an example of the CGSR routing scheme. In CGSR, each node keeps a cluster member table used to map a destination address to the destination 
cluster head address. Each mobile node broadcasts the cluster member table periodically and updates its table after receiving other node broadcasts using the DSDV algorithm. Each node also maintains a routing table that is used to select the next hop to reach the destination cluster.

When receiving a data packet, a node finds the shortest cluster-head along the route to the destination according to the cluster member table and the routing table. Then it refers to its routing table to find the next node in order to reach the selected cluster-head and sends the packet to that node.

The major advantage of CGSR is that only the routes to the cluster heads are maintained and updated. However, this will introduce overhead associated with maintaining clusters and broadcasting cluster tables periodically by each node.

\subsubsection{Global State Routing}

Global State Routing (GSR) is similar to DSDV, with changes to reduce the overhead, which normal DSDV would incur with increasing network sizes. In GSR, each node keeps only its neighbor's link state in its tables. The next hop table and distance table is also kept. The topology table contains the link state information as reported by the destination and the timestamp of the information. The next hop table contains the next hop to which the packets for a destination node should be forwarded. The distance table keeps the shortest distance to each destination node. As in all link state protocols, route update messages are generated upon link change. Upon receiving a routing message, a node updates its topology table if the sequence 
number of the message is higher than the value stored in the table. Fisheye methods, where the approach is to restrict the propagation of topology changes to a subset of the network if the effect is not so profound due to the change, works fine since it will work with both small and large networks. Thus with Fisheye method, nodes keep the most accurate topology information about surrounding regions, and know a little about outer regions. These methods are known to scale well.

\subsubsection{Wireless Routing Protocol}

Wireless Routing Protocol (WRP) [28] is a table-based distance-vector routing protocol. Each node in the network maintains a table with the Distance, Routing, Link-cost, and Message Retransmission List (MRL) information.

The distance table contains the distance of each destination, and the downstream neighbour of each destination and each neighbour. Storing predecessor and successor information in the table is important in detecting loops and avoiding counting-to-infinity problems. The Link-Cost table contains cost of link to each neighbour of the node and the number of timeouts since an error-free message was received from that neighbour. The MRL entries contain the information about the sequence number of a message, a retransmission counter, an acknowledgement-required flag for each neighbor, and a list of updates for the messages. This enables the node to determine when and to whom an update message should be sent. An update message contains information about the destination, the distance to the destination, and the predecessor of the destination. The neighboring 
nodes then modify their tables and try to build new routes through alternate nodes. Consistent check in this manner helps to eliminate looping situations in a better way and also has fast convergence.

The advantage of WRP is that tables guarantee the routes to be optimal, eliminate loops, and achieve fast convergence. However, WRP requires four tables to be stored. Thus, there is a larger memory requirement, compared to other table driven routing protocols.

\subsection{On-demand Routing Protocols}

Source-initiated on-demand routing is a different approach from proactive routing. This protocol is based on a query-reply approach. In contrast to table-driven routing protocols, all up-to-date routes are not maintained at every node. The routes are created as and when required by the source node. When a source node wants to send data packets to a destination, it initiates the route discovery process to find a path to the destination within a network. Once a route has been established, it is maintained by route maintenance mechanisms. The route remains valid until the destination is not reachable or until the route is no longer needed.

\subsubsection{Temporally-Ordered Routing Algorithm}

Temporally Ordered Routing Algorithm (TORA) [27] is a highly adaptive, efficient and scalable distributed routing algorithm based on the concept of link reversal. The basic algorithm is referred to as link reversal algorithm. TORA is designed to minimize reaction to topological changes. The main feature of TORA 
design is that control messages are typically localized to a very small set of nodes. To achieve this, the nodes maintain routing information about adjacent nodes. It guarantees that all routes are loop-free. TORA is proposed for highly dynamic mobile and multi-hop wireless networks. It finds multiple routes from a source node to a destination node.

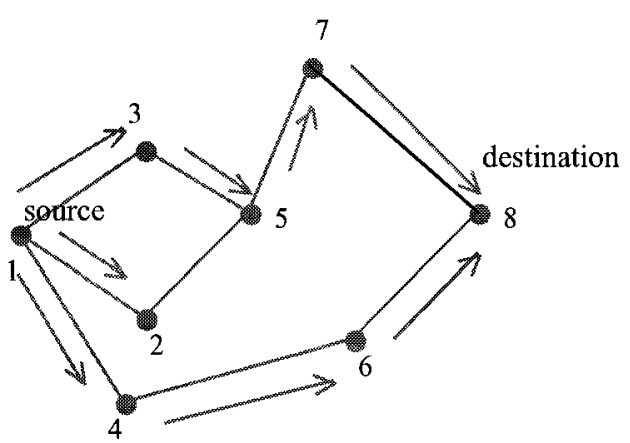

(a) Propogation of QRY message

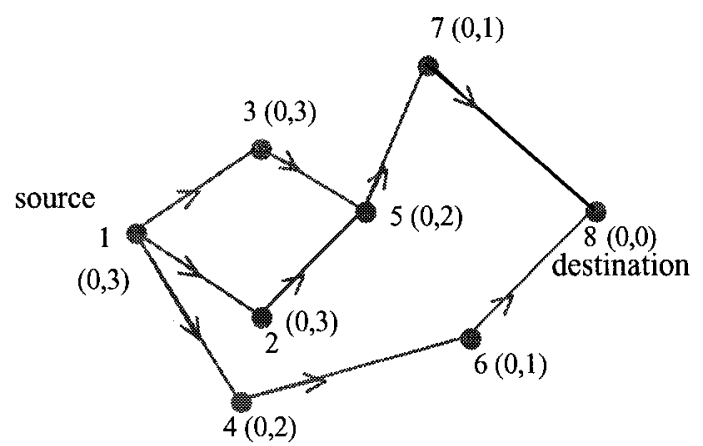

(b) Height of each node updated as a result of UPD

\section{Figure 4. Route creation in TORA}

The protocol can be separated into three basic functions: Route creation, Route maintenance, and Route erasure. Three kinds of packets are used in three functions respectively: query (QRY) for creating route, update (UPD) for both creating and maintaining route, and clear (CLR) for erasing route.

Route Creation is done using QRY and UPD packets. The route creation algorithm uses height metric in which TORA associates a height with each node in the network. The height of destination node is set to 0 and all the other nodes' height is set to NULL. A node with higher height is considered upstream and a node with lower height is as downstream. All messages in the network flow downstream, from 
a node with higher height to a node with lower height. When a node with no downstream link needs a route to a destination, the source broadcasts a QRY packet with the destination node's id. This QRY packet will propagate through the network until it reaches a node that has a route or the destination itself. The non-NULL height node responds with a UPD packet that has its height in it. The node receiving a UPD packet sets its height to one more than that of the node that generated the UPD. In this way directed acyclic graph (DAG) is constructed rooted at the destination. Figure 4 [27] shows route creation in TORA. In Figure 4(b), the source may have received a UPD from node 2 or node 3 , but since node 4 gives it lesser height, it retains that height.

Maintaining routes refers to reacting to topological changes in the network in a manner such that routes to the destination are reestablished within a finite time, meaning that its directed potions return to a destination-oriented graph within a finite time. When a node moves, the DAG route is broken and route maintenance is needed to reestablish a DAG for the same destination. When the last downstream link of a node fails, it creates a new reference level that is higher than any previously defined reference levels. This results in the propagation of that reference level by neighboring. Links are reversed to reflect the change in adapting to the new reference level. This has the same effect as reversing the direction of one or more links when a node has no downstream link.

Upon detection of the network partition, all links in the portion of the network that has become partitioned from the destination are marked as undirected to erase 
invalid routes. The erasure of routes is done using CLR messages. TORA floods a broadcast clear packet (CLR) to erase invalid routes throughout the network.

\subsubsection{Ad-hoc On-Demand Distance Vector Routing}

Ad-hoc On-Demand Distance Vector Routing (AODV) [30] is based on DSDV with some improvement. Without maintaining a complete list of route information in DSDV algorithm, AODV minimizes the number of required broadcasts by creating on-demand routes. AODV nodes use their own sequence number to achieve on-demand mechanism of route discovery and route maintenance. AODV is classified as a pure on-demand route acquisition system because nodes not on a selected path do not participate in route maintenance as routing table exchanges.

When a source node desires to send a message to some destination node and has no route information in its table, it initiates a path discovery process. The source node forwards a route request packet (RREQ) to its neighbors and then RREQ is broadcasted across the network until the destination node or intermediate node with desired route is located. RREQ contains the information of the source node's IP address, current sequence number, broadcast ID and the most recent sequence number. Each node maintains its own sequence number and broadcast ID. The broadcast ID, which is incremented for every RREQ, and together with the node's IP address, uniquely identifies a RREQ. A node discards a route request packet that it has already seen. The route request packet uses sequence numbers to ensure that 
routes are loop free and to make sure that if intermediate nodes reply to route requests, they only reply with the latest information. After receiving a RREQ, a node may send a route reply (RREP), if it is either the destination or if it has an active route to the destination with corresponding sequence number greater than or equal to that in the RREQ. Then it forwards route reply packet back to the source node. Otherwise, it will broadcast RREQ to its neighbours. As the RREP propagates back to the source node, intermediate nodes create forward pointers to the destination. The node receiving the route reply packet increments the hop count by one and updates routing information for the destination node in the routing table, then builds the forwarding route to the destination. Once the RREP reaches the source node, the source can forward packets along the discovered route. When RREP is sent back to source, it is likely that the source node or intermediate nodes may receive the same RREP packet more than once. The same RREP may contain greater sequence number or contains the same sequence number with a smaller hop count. In this case, this node may update its routing information for that destination and use the better route.

As long as the route remains active, it will continue to be maintained. A route is considered active as long as there are data packets periodically traveling from the source to the destination along that path. Once the source stops sending data packets, the links will time out and eventually be deleted from the intermediate node routing tables. If a link break occurs while the route is active, the node upstream of the break propagates a route error (RERR) message to the source node to inform it 
of the new unreachable destination. After receiving the RERR, if the source node still desires a route, it can reinitiate route discovery.

\subsubsection{Associativity-Based Routing}

Associativity-Based Routing (ABR) [36] protocol is uniform and destinationbased protocol, it is free from loops, deadlock, and packet duplicates. ABR defines a

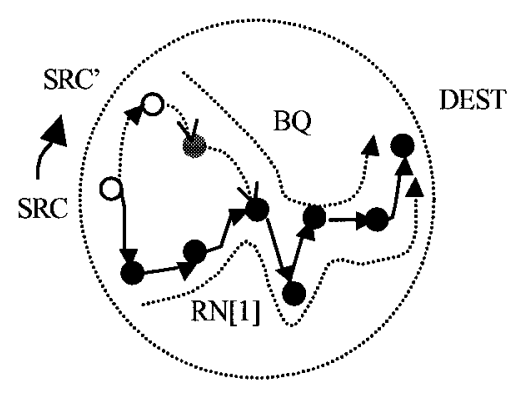

(a) Route Maintenance for a Source Move

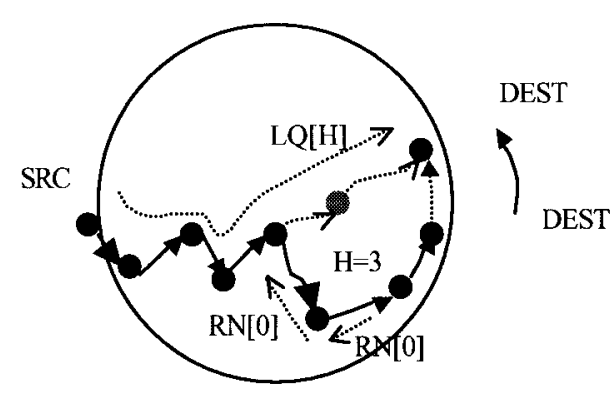

(b) Route Maintenance for a Destination Move

\section{Figure 5. Route maintenance for source and destination movement in ABR}

new routing metric for ad-hoc mobile networks, by which ABR uses end-to-end topology information in route selection.

This metric is known as the degree of association stability. In ABR, a route is selected based on the degree of association stability of mobile nodes. Each node periodically generates a beacon to signify its existence. After receiving the beacon, neighboring nodes update the associativity tables. For each beacon received, the associativity tick of the current node with respect to the beaconing node is incremented. Association stability is defined by connection stability of one node with respect to another node. The node with high degree of association stability has a low mobility, otherwise has high mobility. Associativity ticks are reset when the 
neighbors of a node or the node itself moves out of range. A basic objective of ABR is to derive longer-lived routes for ad-hoc mobile networks.

Three phases of ABR are: (a) route discovery, (b) route reconstruction (RRC), and (c) route deletion. Route discovery is as follows: The route discovery phase is accomplished by a broadcast query and await-reply (BQ-REPLY) cycle. A node desiring a route broadcasts a BQ message in search of mobiles that have a route to the destination. All nodes (except for destination node) receiving the query message append their addresses and their associativity ticks with their neighbors to the query packet. A successor node erases its upstream node neighbors' associativity tick entries and retains only the entry concerned with itself and its upstream node. In this way, each resultant packet arriving at the destination will contain the associativity ticks of the nodes along the route to the destination. The destination is then able to select the optimal route by examining associativity ticks along each of paths. In the case where multiple paths have the same overall degree of association stability, the route with the minimum number of hops is selected. The destination then sends a REPLY packet back to the source along this path. Nodes propagating the REPLY mark their routes as valid. All other routes remain inactive and the possibility of duplicate packets arriving at the destination is avoided. Route reconstruction may consist of partial route discovery, invalid route erasure, valid route updates, and new route discovery, depending on which node along the route moves. Movement by the source results in a new BQ-REPLY process, as shown in Figure 5a [36]. RN[1] message is a route notification that is used to erase the route entries associated with 
downstream nodes. When the destination node moves, the immediate upstream node erases its route and determines if the node is still reachable by a localized query (LQ[H]) process, where $\mathrm{H}$ refers to the hop count from the upstream node to the destination. If the destination receives the LQ packet, it replies with the best partial route; otherwise, the initiating node times out and the process backtracks to the next upstream node. Here an RN[0] message is sent to the next upstream node to erase the invalid routes and inform this node that it should invoke a $L Q[H]$ process. If this process results in backtracking more than halfway to the source, the LQ process is discontinued and a new BQ process is initiated at the source.

When a discovered route is no longer desired, the source node initiates a route delete (RD) broadcast so that all nodes along the route update their routing tables. The RD message is propagated by a full broadcast, as opposed to a directed broadcast, because the source node may not be aware of any route node changes that occurred during route reconstructions.

\subsubsection{Location Aided Routing}

Location Aided Routing (LAR) is a variant of the DSR routing scheme and works on demand. The aim of the protocol is to limit route discovery flooding, so as to reduce control packet overhead. It accomplishes this by using GPS data. Using this information, flooding is done within a defined rectangular region. Mobile nodes here can also store the speed of the target node, so that it can predict where the target node will possibly be at a later point of time. 
A distinguishing characteristic of these location-based protocols is that, to forward packets, a node only requires three pieces of information, that of its own position, that of the destination, and those of its adjacent neighbors. A transmitting node uses a location service to determine the location of the destination, and includes this information as part of the destination address in its messages. Routes do not need to be established or maintained explicitly; thus, there is no need to store routing tables at the nodes, and no need for routing table updates. Typically adjacent nodes are identified by broadcasting limited range beaconing messages and by employing various time-stamping mechanisms. The beaconing message includes distance limits. A receiving node discards this message if its location lies beyond the distance limit.

The availability of accurate location information at each node is essential for location-based routing to work, which, in turn, requires timely and reliable location updates as nodes change their locations. One or more nodes, designated to act as location servers, coordinate these location service functions, which are necessarily decentralized because of mobility. Many of the ongoing researches focus on designing efficient location services. 


\section{Chapter 3}

\section{Energy-related metrics in network protocol}

Wireless ad hoc network is a system of autonomous mobile nodes that cooperatively route packets for each other. In such a network, nodes other than the packet source and destination participate in the delivery process. The thesis is primarily concerned with ad hoc networks where energy consumption is an issue, for instance because nodes are battery-powered. Battery technology is not improving at a rapid rate, so power consumption is likely to remain an issue in mobile wireless networks.

One important characteristic of wireless ad hoc networks is that nodes are energy-constrained. Because MANET [22] nodes are always very small, battery power and energy management system are critical issues in building these networks. Nodes are battery-operated and frequent recharging or replacement of batteries may be undesirable or even impossible. This makes energy-efficiency an important metric in wireless protocols. Since power is often an important issue in many ad hoc networks, there have been many proposals to incorporate power-awareness into different routing layers. Most recently, several efforts have focused on building power-aware protocols in MANET. Some routing algorithms in [41] concentrate on efficient power utilization. This thesis is trying to maximize the lifetime of the whole network by evenly consuming the energy of each node. In routing protocol development, the optimal protocol should consider some reasonable metrics 
simultaneously. The metrics worthy of considerations are as follows: throughput, minimum delay, overhead, load balancing, adaptability to the changing topology, association stability (longevity of routes), and total power consumption. Data throughput and delay are statistical measures of data routing performance (e.g., means, variances, distributions) [22]. These are the measures of a routing policy's effectiveness as measured from the external perspective of other policies that make use of routing [22]. Overhead is an important metric. By minimizing the communication overhead, a protocol can conserve available bandwidth and increase the adaptability. Load balance is a critical issue in building protocols. In a network, a node always in an advantageous position can communicate with most of nodes. Some of current protocols seem to favor routing traffic through this node, thereby causing a bottleneck. Because of the mobility characteristic of MANET, it is important for a protocol to discover long-lived routes by considering the topology change. In the power-aware network, minimum total power is one of the critical factors because of energy limitation. In section 3.1, we discuss details in some power-aware metrics.

\subsection{Energy Efficient Metrics}

Many researches focus on designing protocols to reduce energy consumption, increasing the lifetime of each node, maximizing the lifetime of the whole network by evenly distributing the power consumption rate of each node, and minimizing the overall transmission power for the connection request. Two constraints should be 
satisfied simultaneously in the optimal protocol. Since the network topology is dynamically changing, bandwidth and battery power are significant factors in the network. There exists trade-offs in using different metrics beyond the end-to-end delay. Therefore, in routing protocol design, one should optimize some reasonable metrics in addition to others. Here is a list of metrics defined to study the performance of power-aware routing protocols: energy consumed per packet, network lifetime (time to network partition), power variance among mobile nodes, cost per packet, maximum mobile cost, maximum end-to-end throughput, minimum end-to-end delay, minimum total power, least congest path, minimum overhead, adaptability to the changing topology, longevity of routes, and route relaying load. My thesis mostly concentrates on the first three metrics.

\subsubsection{Maximize network lifetime (Time to network partition)}

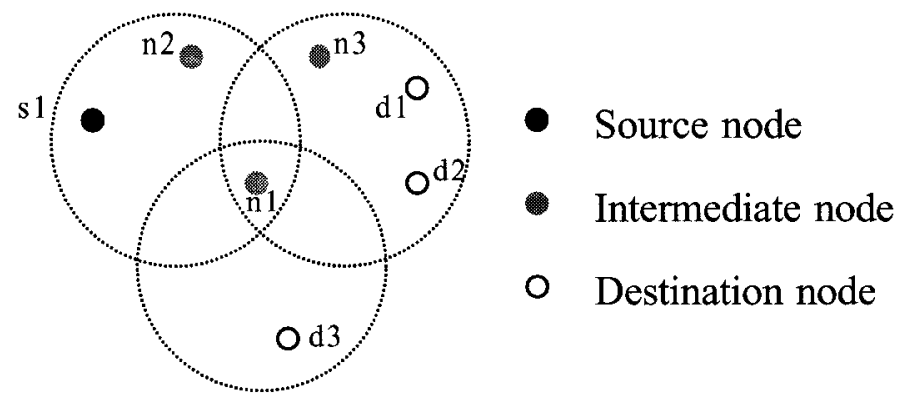

Figure 6. Mobile network communication

In a network topology, network lifetime is the time until removal of a small set of nodes will cause the network to partition. The connection of the nodes in the two partitions must go through some of the critical nodes. To prevent the network 
partition, these critical nodes should not be overused. Therefore, a routing algorithm should avoid partition of the network by using nodes' energy evenly. In Figure 6, when the source node sends the packets to destination nodes $\mathrm{d} 1$ and $\mathrm{d} 2$, it always uses the critical node nl by applying shortest hops algorithm in many protocols. This will leads to the energy depletion of node $\mathrm{n} 1$ and result in the partition of the network. After that, if $\mathrm{s} 1$ sends packets to $\mathrm{d} 3$, it will need $\mathrm{n} 1$ as an intermediate node. But $\mathrm{n} 1$ is not valid because of power shortage. To avoid this, the source uses other two paths to destinations $\mathrm{d} 1$ and $\mathrm{d} 2, \mathrm{~s} 1-\mathrm{n} 2-\mathrm{n} 3-\mathrm{d} 2$ and $\mathrm{s} 1-\mathrm{n} 2-\mathrm{n} 3-\mathrm{d} 3$. Although it can increase the total transmission energy, it prevents network partition.

\subsubsection{Minimize power variance among mobile nodes}

The main idea of this metric is that routing algorithm assigns the fair energy distribution among the nodes. In this way, all mobiles are equal and no one mobile is penalized or privileged over any other. When a protocol minimizes power variance among mobile nodes, it can maximize the network lifetime. This metric ensures that all nodes in the network remain up and running together for as long as possible. In Figure 6, by using the two substitute routes, the energy level difference among nodes $\mathrm{n} 2, \mathrm{n} 3$, and $\mathrm{n} 1$ can be reduced.

\subsubsection{Minimize energy consumption per packet}

The total energy of sending data packets along some route is the sum of node energy consumption. When energy consumption per packet is minimized, the total 
energy consumption is also minimized. Under light loads, this metric will most likely result in the shortest-hop path. If we try to derive a routing algorithm that maximizes the network lifetime, we need to minimize the cost per packet. When using these metrics, routes should be created such that mobile nodes with little energy reserve are not on many routes. As network load increases, this metric is not necessary any longer because it will only transmit packets around congestion areas in the network.

\subsubsection{Minimize maximum node cost}

This metric attempts to minimize the cost consumed by a mobile node when forwarding a data packet through it. Minimizing the cost per node results in significant reduction in the maximum node cost. Moreover, network transmission failure is delayed and energy variance among nodes is reduced in this metric.

As a result of applying the above metrics, a shortest-hop (or shortest-path) routing protocol may no longer be applicable. However, a shortest cost routing protocol with respect to the above energy efficiency metrics would be necessary. During the transmission of packets, a cost function may be used to accurately reflect the remaining lifetime of each node. The premise behind this approach is that although packets may be routed through longer paths, the paths contain nodes that have more energy reserve. Also, energy can be conserved by routing traffic through lightly loaded nodes because the energy used in contention and retransmission is minimized. 
The advantage of power-aware routing metrics and the effect of the metrics on end-to-end delay are studied in [37] by Singh. He compared shortest hop routing with the power-aware shortest cost routing schemes in the performance of delay, average cost per packet, and average maximum node cost. The simulation results show that power-aware routing scheme does not result in more delay than the traditional shortest path routing algorithm because power-aware metric often avoids congested paths. However, there is significant improvement in average cost per packet and average maximum node cost in which the cost is in terms of the energy efficient metrics defined above. Improvements are substantial for large networks and heavily loaded networks. Therefore, by adjusting routing parameters, a more energy efficient routing scheme may be utilized for wireless networks. The above approach to routing in wireless ad hoc networks requires that every mobile have knowledge of the locations of every other mobile and the links between them. This creates significant communication overhead and increased delay. Research completed in [38] addresses this issue by proposing localized routing algorithms, which depend only on information about the source location, the location of neighbors, and location of the destination. This information is collected through GPS receivers, which are included within each mobile. Therefore, excessive network communication is not required. A new power-cost metric incorporating both a mobile's lifetime and distance based power metric is proposed, and using the newly defined metric, three power-aware localized routing algorithms are 
developed: power, cost, and power cost. The power algorithm attempts to minimize the total amount of power utilized when transmitting a packet, whereas the cost algorithm avoids mobiles that maintain low battery reserves in order to extend the network lifetime. Finally, the power-cost routing algorithm is a combination of two algorithms. Experiments validated the performance of these routing algorithms.

\subsection{Classification of Conserving Energy Routing Protocols}

There is rich research in conserving energy routing protocols. After a detailed analysis, the mechanism can be divided into three different groups: switching on/off transmitters to save energy, routing based on metrics of energy consumption, and network topology control by adaptively adjusting transmitter radios.

\subsubsection{Switching on/off transmitters to save energy}

Network nodes have three states: sleeping, listening and active. Radios consume energy not only when transmitting and receiving packets, but also when the node is idle. Nodes will waste much power by listening to other nodes. Then a lot of energy could be saved through turning off the radio of idle nodes. Authors in [45] proposed mainly two routing algorithms, which can trade off energy dissipation and data delivery quality according to application requirements. They build the routing algorithm based on the existing on-demand ad hoc routing protocols including AODV and DSR, without changing the underlying routing protocols. Radio of the idle nodes is turned off to reduce power consumption with the involvement of application-level information and additional use of node deployment density to 
adaptively adjust routing fidelity to extend the whole network lifetime. Simulation studies [45] show that this algorithm can save up to $50 \%$ of node's energy. And it also shows that greater node density can be used to increase network lifetime.

The two routing algorithms presented in [45] are AFECA (Adaptive Fidelity Energy-conserving Algorithm) and BECA (Basic Energy-conserving Algorithm). AFECA algorithm makes best use of the interchange among nodes in ad hoc networks. AFECA demonstrates adaptive fidelity. It adapts sleep times based on node density if many interchangeable nodes are present. AFECA improves energy conservation by estimating node populating and increasing sleep time when other active nodes are available. This routing algorithm saves energy by identifying nodes that are equivalent from a routing perspective and then turning off unnecessary nodes, keeping a constant level of routing fidelity, which is defined as uninterrupted connectivity between communicating nodes.

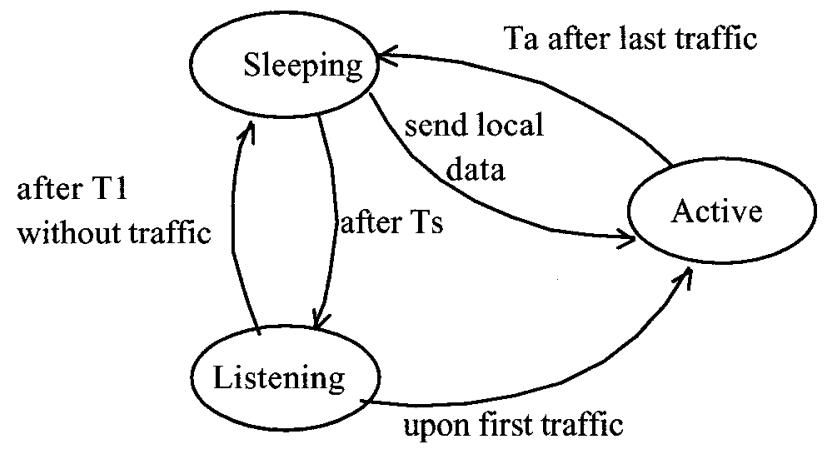

\section{Figure 7. Three states in BECF}

Figure 7 illustrates the three states in BECF [45]. Initially the node begins at the sleeping state and its radio is off. The node keeps the sleeping state for time Ts, and 
then switches to listening. When sleeping, if the node has data packet to send, it switches to active state and starts to forward data packet. At this time its radio is still off, but other sensors or parts of the node may be on. In listening state, a node turns on its radio and listens for messages for time Tl. After receiving a routing message, it will participate in the routing. When it decides to transmit data packets, it switches the state to active. Otherwise it returns to sleeping after $\mathrm{Tl}$. When in active state, a node sends or transmits data packets. If at any time it no longer has data to send or relay during $\mathrm{Ta}$, it switches to sleeping state and turns radio off.

\subsubsection{Topology control by adaptively adjusting radio power}

Topology is an important issue especially in mobile ad hoc networks. The topology of a multi-hop wireless network is the set of communication links between node pairs. In [33], authors approach the energy efficient problem by controlling the network topology. Little has been done in the area of adjusting controllable parameters in order to create the desired topology. In their work, they proposed two centralized algorithms for static networks, which are shown to be optimal, and two distributed heuristics that adjust nodes. The topology need to be controlled because the wrong topology can considerably reduce the capacity, increase the end-to-end delay, and decrease the robustness to node failures. Sparse networks can cause network partition and high end-to-end delay, while dense networks can cause the limited spatial reuse to reduce network capacity. The battery life of nodes can be extended by using transmit power control. No research has considered the 
assignment of different transmit powers to different nodes to meet a global topological property, such as a connected network and studied the implementation in the context of a typical ad hoc network.

\subsubsection{Routing based on the metric of energy consumption}

\subsubsection{Minimum Total Transmission Power Routing}

MTPR [40] algorithm is focusing on minimizing the total transmission power consumed without considering the remaining energy of each node. MTPR minimizes the total energy consumption as the packets are transmitted through the selected route. MTPR does not maximize the mobile network lifetime. Because the residual energy of mobile nodes is not taken into account, nodes on the desirable route will suffer early failure due to their heavy transmission load.

MTPR algorithm uses a simple energy metric, which represents the total energy consumed along the transmission route. This algorithm can be applied to a standard shortest path algorithm such as Dijkstra or Bellman-Ford. We consider a generic route

$$
r_{d}=n_{0}, n_{l}, \ldots, n_{d}
$$

Where $n_{0}$ is the source node and $n_{d}$ is the destination node.

An energy function $P\left(n_{i}, n_{i+1}\right)$ represents the transmission energy over the hop $n_{i}$ and $n_{i+l}$ and the total transmission power for the route $l$ is calculated as:

$$
P_{l}=\Sigma P\left(n_{i}, n_{i+1}\right), \text { for all nodes in the route, where } 0 \leq i \leq d-1 .
$$


Therefore the desired route $k$ can be derived from

$$
P_{k}=\min P_{l} \text {, where } l \in \mathrm{A}, \mathrm{A} \text { is the set containing all possible routes. }
$$

In [40], the transmission range is fixed to 250 meters. Hence, it means that MTPR chooses the shortest path among the possible routes, thus behaves like the protocol using shortest-hop route. Only when all nodes can adjust their transmission ranges according to the distance between nodes, MTPR can reduce the total transmission power by selecting routes with more hops having short transmission ranges.

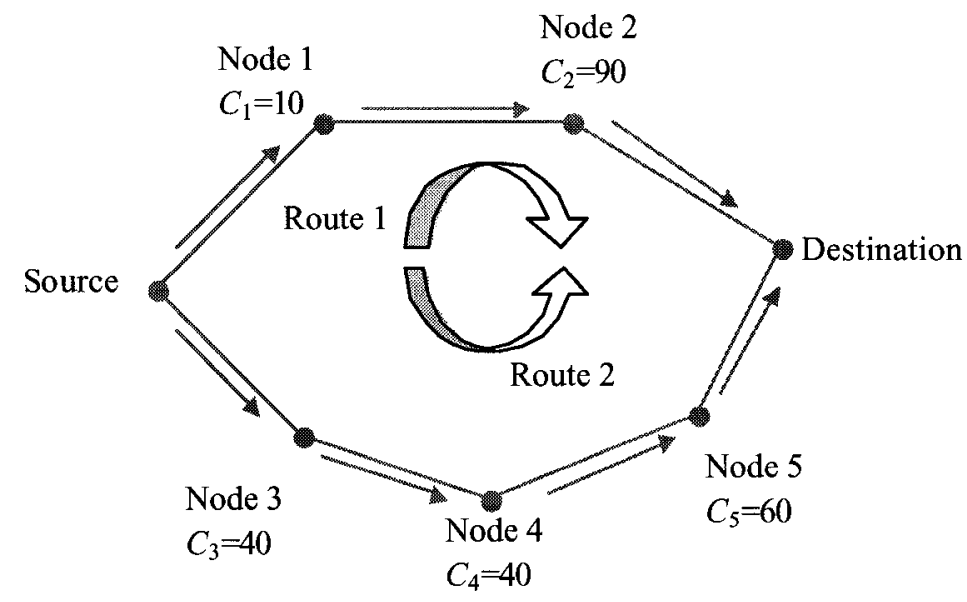

Figure 8. An illustration of route selection using different algorithms

Since transmission power depends on distances, such as the transmission power is proportional to $d^{\alpha}$, where $d$ is the distance between two nodes, MTPR prefers routes with more hops having short transmission ranges to those with fewer hops but having long transmission ranges. The more nodes involved in forwarding packets, the greater the end-to-end delay. Moreover, a route with more nodes is more likely to be unstable, because the probability that intermediate nodes will move out of 
range is higher. However, transceiver power as well as transmission power can also be considered as a cost metric. This will help the algorithm to find routes with fewer hops because generally the transceiver power is identical for hosts. This routing algorithm guarantees the lowest transmission power in each session. Because MTPR does not consider the remaining power of each node, it might not succeed in extending the lifetime of each host.

In Figure 8, C represents the power of each node. By using MTPR, if the transmission energy is same for each transit, route 1 should be selected as the desired route since there are fewer nodes in route 1 and then less transmission energy than route 2. However, if the transmission energy for each operation is greater than 10 , it could not work well since the energy of node 1 is less than transmission power.

\subsubsection{Minimum Battery Cost Routing}

The fatal disadvantage in MTRP is only considering the total transmission power, since it does not reflect directly the lifetime of each node. If a specific host is always on the minimum total transmission power route, the battery power of this node will be exhausted quickly. To prevent this, the remaining battery capacity of each node is a more accurate metric to define the lifetime of each node.

MBCR [40] algorithm is described as follows:

$c_{i}(t)$ : battery capacity of node $\mathrm{n}_{i}$ at time $t$. 
A battery cost function $f_{i}\left(c_{i}\right)$ can be defined according to the probability to forward packets. The function $f_{i}\left(c_{i}\right)$ is possible to choose

$$
f_{i}\left(c_{i}\right)=\frac{1}{c_{i}(t)} \text {, where } c_{i}(t) \text { is the battery capacity of node } i \text { at time } t \text {. }
$$

The less capacity a node has, the more reluctant it is to forward packets.

Then the cost $R_{j}$ for route $j$ is

$$
R_{j}=\Sigma f_{i}\left(c_{i}\right) \text {, where } n_{i} \text { is the node along route } j
$$

In MBCR, the optimal route $k$ satisfies

$$
R_{k}=\min R_{j} \text {, where } j \in \mathrm{A}, \mathrm{A} \text { is the set containing all possible routes. }
$$

In Figure 8, route 2 will be selected according to MBCR since the cost of route 1 is 0.11 and the cost of route 2 is 0.07 .

Since node battery capacity is directly incorporated into the route selection algorithm, MBCR algorithm prevents specific sets of nodes from being overused, thereby increasing node lifetime and the network lifetime. Minimum cost routing algorithm minimizes the total cost of packets transmission. The shape of the cost function controls the extent to which the presence of a high cost node on a route deflects traffic from that route. It is a critical step to select the desirable cost function when developing the minimum energy routing protocol. 


\subsubsection{Min-Max Battery Cost Routing}

In MBCR, because only the total battery cost function is considered, a route containing nodes with little remaining battery may still be selected. To make sure to avoid overusing specific nodes, S. Singh et al. proposed MMBCR [40], in which the cost function can be modified to avoid selecting route with nodes having the least battery capacity among the nodes in all possible routes. MMBCR allows the nodes with high residual power to participate in the routing process more often than the nodes with low residual power. The battery will be used more fairly.

The MMBCR algorithm is as follows:

MMBCR defines the route cost as

$R_{j}=\max f_{i}\left(c_{i}\right)$, where node $i$ is in the set of all nodes along route $j$.

The desired route $k$ satisfies

$$
R_{k}=\min R_{j} \text {, where } j \text { is the set of all possible routes. }
$$

In Figure 8, route 2 will be selected by using MMBCR . In this network, route cost of route 1 is 0.1 and that of route 2 is 0.025 . The route with less cost will be chose. Obviously, MMBCR is better than MTPR if the transmission energy is very large. This algorithm cannot guarantee that minimum total transmission power path will be selected under all circumstances. It can consume more power to transmit packet from a source to a destination Since MMBCR considers the weakest and crucial node over the path, a route with the best condition among paths impacted by each crucial node over each path is selected. 


\subsubsection{Conditional Min-Max Battery Cost Routing}

To maximize the lifetime of each node and avoid overusing some nodes, we cannot achieve this only by applying MTPR or MMBCR. To solve this problem, battery capacity instead of cost function is used as a route selection metric. Conditional Min-Max Battery Cost Routing (CMMBCR) [40] is the solution, which is combination of MTPR and MMBCR. In CMMBCR, the algorithm defines the battery capacity for route $R_{j}$ at time $t$ as

$$
R_{j}(t)=\min c_{i}(t) \text {, where node } i \text { is in the set of all nodes along route } j .
$$

For route selection, we consider two sets $\mathrm{Q}$ and $\mathrm{A}$, where $\mathrm{Q}$ is the set of all possible routes and $\mathrm{A}$ is the set of all possible routes with

$$
R_{j}(t) \geq \gamma \text {, where } \gamma \text { is fixed threshold. }
$$

The route selection algorithm is as follows: if all nodes in a given paths have remaining battery capacity higher than $\gamma$, choose a path in $\mathrm{A} \cap \mathrm{Q} !=\varnothing$ by applying MTPR; otherwise select a route $R$ with the maximum battery capacity by applying MMBCR. In Figure 8, by using CMMBCR, if the threshold $\gamma$ is set to 5, route 1 is the desired route. Otherwise, if the threshold $\gamma$ is set to 11 , the source will choose route 2 to send packets. This shows that threshold $\gamma$ is a very important factor for route selection in this algorithm.

CMMBCR is an algorithm that when all nodes in some possible routes have sufficient remaining battery capacity, a route with minimum total transmission 
power is chosen. Since less total power is required to forward packets for each connection, the relaying load for most nodes will be reduced and their lifetime will be extended. However, if all routes have nodes with low battery capacity, routes including nodes with the lowest battery capacity should be avoided to extend the lifetime of these nodes. So it is clear that the threshold can be viewed as a protection margin. If some nodes' battery capacity goes below this value, they will be avoided to elongate the lifetime. The performance of CMMBCR will therefore depend on this value. The CMMBCR considers both the total transmission energy consumption of routes and the remaining power of nodes.

In [18], the author compared the performance of MTPR, MBCR, and MMBCR according to network density. In dense networks, because of the availability of several routes, it seems to be more important to reduce the overall energy consumption so as to prolong the lifetime of each individual node [18]. As for sparse networks, the role of nodes for avoiding network partition is more crucial [18]. In Chapter 5, we compare EPDSR with MTPR, MBCR, and MMBCR. 


\section{Chapter 4}

\section{DSR Protocol description and Implementation in NS2}

DSR [15] is a popular on-demand protocol. Network simulator NS2 was used in our simulations. We modified DSR protocol to implement the energy efficient protocol EPDSR and compared performance of these two protocols in three metrics. Before introducing EPDSR, we need more knowledge about DSR and simulator tool NS2. NS2 is developed by VINT project at University of California at Berkeley and extended with the simulation of multihop wireless networks by the MONARCH research groups at Carnegie-Mellon University. Our simulations are based on NS2 version $2.1 \mathrm{~b} 9 \mathrm{a}$ under windows.

\subsection{DSR Protocol}

\subsubsection{Protocol description}

DSR protocol is an on-demand routing protocol that is based on the concept of source routing. DSR is a simple and efficient routing protocol designed specifically for use in multi-hop wireless ad hoc networks of mobile nodes. Without infrastructure or administration, the network with DSR protocol is completely selforganized and self-configured. Mobile nodes in the network maintain route caches containing source routes. Nodes update the entries information in the route cache if 
a better route is found, when it learns about new routes. For the mobile nodes, the cooperation to forward data packets to allow communication through multiple nodes is not directly. The data transmission always needs several hops to forward from the source to the destination since each node has limited transmission range.

DSR allows nodes to dynamically discover a route across multiple network hops to any destination in the network. DSR requires that each packet keep its route information, thus eliminating the need for every node in the network to do periodic route discovery advertisements. DSR performs a route discovery and takes required actions for maintaining that route.

DSR protocol consists of two major phases: route discovery and route maintenance.

\section{Route discovery:}

Mobile nodes in ad hoc networks use route discovery process to dynamically discover a route to any other node in the network, no matter directly reachable in the communication range or reachable through other intermediate network hops. Route discovery works by flooding a request through the network in a controlled manner, seeking a route to some target destination [24]. In Figure 9 [36], we demonstrate the packet broadcasting through source $\mathrm{N} 1$ to destination $\mathrm{N} 8$. When a mobile source node has a packet to send to some destination, it first consults its route cache to determine whether it already has a route to the destination. If it has a route to the destination, it will use this route to send the packet. On the other hand, if the node 
does not have such a route, it initiates RREQ (route request packet), which may be received by the nodes within the wireless transmission range. RREQ message identifies the source and the destination for route discovery. This route request message contains the address of the destination node, along with the source node's address and a unique identification number determined by the initiator of the request message. The RREQ message also records the address of each intermediate node through which this route request message has been forwarded. After receiving the packet, the node checks whether there exists a route to the destination. If there does not, the node appends its own address to the route record of the packet and broadcasts it with the same identification number value to all its neighbors.

To limit the number of route requests propagated on the outgoing links of a node, a node only forwards the route request message which has not yet been seen by this node and if the node's address does not already list in the route record.

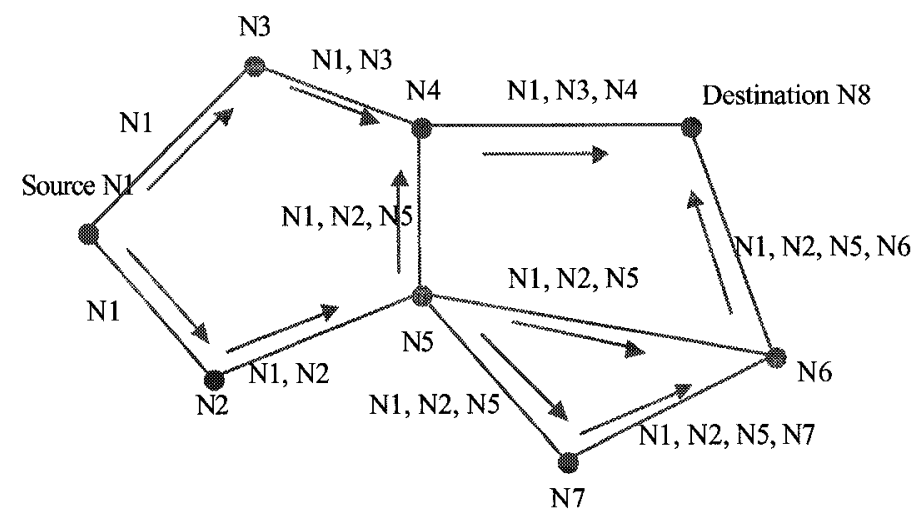

Figure 9. Route discovery in DSR: broadcasting RREQ 
A route reply is generated when the route request reaches either the destination itself or an intermediate node that contains a route to the destination in its route cache. RREP is only replied by the destination or an intermediate node that knows the route. RREP has the copy of the accumulated route record from the RREQ. Figure 10 [36] illustrates the formation of the route record as the route reply propagates throughout the network.

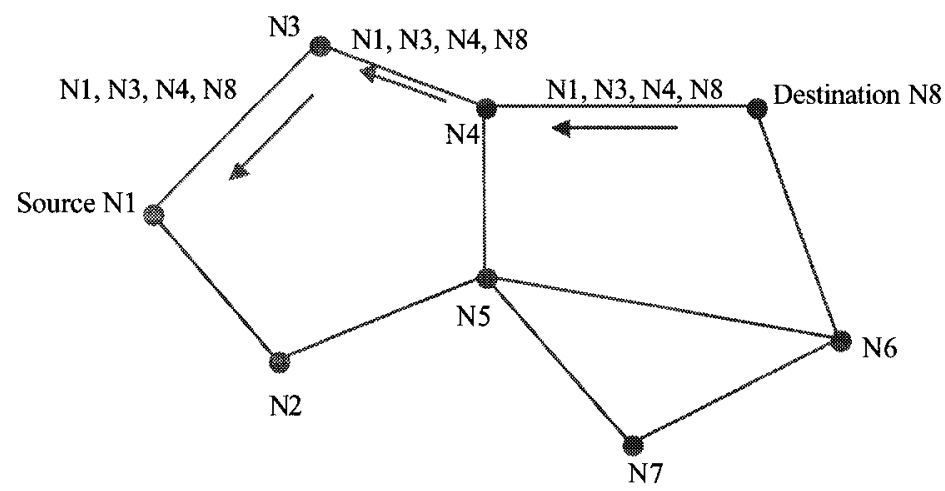

Figure 10. Route discovery in DSR: propagation of the route reply

If the destination node generates the route reply message, it places the route record contained in the route request into RREP. If RREP is generated by the intermediate node, this node will append its cached route to the route record and then generate the route reply. To return the route reply, the responding node, which generates the RREP message, must have a route to the source. If it has a route to the source in its route cache, it may use that route. Otherwise, if symmetric links are supported, the node may reverse the route in the route record. If symmetric links are not supported, the node may initiate its own route discovery and piggyback the route reply on the new route request. The selected return route may either be a list reversal 
that is piggybacking or using another existing route in the destination node's table. Thus the route may be considered unidirectional or bi-directional. DSR nodes stay awake and listen everything importance to their routing tables in promiscuous node, so that route discovery may speed up.

\section{Route Maintenance:}

Route maintenance is accomplished through the use of two types of packets, RERRs (route error packets) and ACKs (acknowledgments). Whenever a node encounters fatal transmission errors so that the route becomes invalid, the Error RERR packet is generated, and is forwarded to the source node. When a route error packet is received, the node in error is removed from the node's route cache and all routes containing this node are truncated at that point. Source node removes the erroneous hop from all of its route cache entries, and selects a new route, or if there are no more available routes, it initiates a new route discovery.

ACK packets are used to verify the correct operation of the route links. There is also a passive acknowledgement when a node hears after forwarding the packet along the route. DSR mainly relies on MAC for notification of link failures. Some researches give us a detailed study on on-demand behaviour of DSR, in which protocol's forwarding latency and route caching correctness are analyzed. These study indicate that DSR, using its route-cache wisely, can greatly reduce the overhead of its control messages. 


\subsection{Simulation Setup and System Parameters}

NS2 [17] is a discrete event simulator targeted at networking research. It provides support for wired and wireless networking with multicast capabilities and satellite networks. It provides substantial support for simulation of TCP, routing, unicast and multicast protocols.

NS2 is written in $\mathrm{C}++$ and OTCL script language based on Tc1/Tk. NS2 users write an OTCL script that defines the node number and links in the network, the traffic pattern, and the protocol that they use. NS2 uses this script to do the simulation. The result of the simulation is an output trace file, which is used to do the data analysis, delay and throughput calculation, power consumption and other metrics, and to visualize the result using network animator (NAM). NS2 is developed by VINT project at University of California at Berkeley, and the original NS does not support wireless environment. Current NS2 with the wireless mobility extension is developed by Monarch research groups at Carnegie-Mellon University. NS2 simulator supports for simulation of multihop wireless networks completely with physical, data link, and medium access control (MAC) layer models. However, this work still is not completed. Researchers keep on working it to develop more protocols and solve the bugs there.

In this thesis, we use the Monarch extension since it supports ad hoc networks. It is open source, and can be downloaded from the Internet. It provides tools for generating data traffic and mobility scenario patterns for the simulation. 
Four ad hoc network routing protocols AODV, DSDV, DSR and TORA have been implemented in NS2. The Monarch extension NS2, along with the original NS, has the following features.

\subsubsection{Radio Propagation Models:}

Propagation models are used to determine if the data transmitted through the air has been successfully received. These models also consider propagation delay and capture effect and carrier sense.

Two Ray Ground Reflection Approximation uses Friss-space attenuation $\left(d^{2}\right)$ at near

distances and an approximation to Two Ray Ground $\left(d^{4}\right)$ at far distances. The approximation assumes specula reflection off a flat ground plane. The antenna is an omnidirectional antenna.

\subsubsection{Media Access Control Protocols:}

The IEEE 802.11 Distributed Coordination Function (DCF) MAC protocol is used in the simulation of NS. MAC layer handles collision detection, fragmentation and acknowledgements. This protocol may be used to detect the transmission error. The simulator uses the "RTS/CTS/Data/ACK" pattern for unicast packets and a "Data" pattern for broadcasting packets. 802.11 avoids collisions by checking the channel before using it. If the channel is available, it can begin to transmit the packets. Otherwise it must wait a random amount of time before resending. For each retry, exponential backoff algorithm is used. In a wireless network, we cannot 
assume that all stations hear each other. If a station senses the medium free, it may not necessarily be so. This problem is called as hidden terminal problem and to overcome these problems, the collision avoidance mechanism and positive acknowledgement scheme are used together. Positive acknowledgement requires a node send an acknowledgement when it receives a data packet. The sender will continue to retransmit this packet until it receives the acknowledgement message or the number of the retransmission exceeds the limits.

\subsubsection{Mobile nodes:}

Mobility support is one of important characteristics of Monarch Project. Mobile node is the basic object with added functionalities, which can make movement, receive, and transmit on a channel. Mobility features include node movement, periodic position updates, and maintenance of topology boundary.

Each mobile node is attached a routing agent for calculating routes to other nodes in the network. The agent determines a routing path for the packet and stamps it. It forwards the packet down to the link layer. The link layer uses ARP to determine the hardware addresses of neighbouring nodes and maps IP addresses to their correct interfaces. The packet is then sent to the interface queue, and stays there until a signal from MAC is received. It leaves the IFQ and waits for MAC to send it when the channel is available. The packet is copied to all interfaces at the time when the first bit of the packet would begin arriving at the interface in a real physical system. Each network interface stamps the packet with its own properties, 
and invokes a propagation model. Note that the propagation model is invoked at the received part. This propagation model uses transmit and receive stamps to determine the power that the interface will receive the packet. The receiving network interface is left to decide whether the packet is received successfully or not. If successful, the packet is passed to MAC layer. If MAC layer receives this packet as error-free and collision-free, it passes the packet to node's entry point. The packet then reaches a demultiplexer, which decides whether packet should be forwarded again or if it has reached its destination node. If arrived point is the destination node, packet is sent to the demultiplexer, which decides the application that should be delivered. If the packet is forwarded, this operation is repeated.

\subsection{Simulation of DSR in NS2}

We use the simulation tool NS2 to simulate DSR and EPDSR for different scenarios, and use three performance metrics to evaluate the average performance of two protocols.

\begin{tabular}{|l|l|}
\hline Number of nodes & 50 \\
\hline Dimension of simulation area & $1500 \mathrm{~m} * 300 \mathrm{~m}$ \\
\hline Initial node energy (Joules) & 100 \\
\hline Simulation time (seconds) & 1000 \\
\hline Traffic type & CBR, 4 packets/s \\
\hline Packet size (bytes) & Various, 64 1024 \\
\hline Number of sources & 14 \\
\hline Number of connection nodes & 20 \\
\hline Mobility Model & Random Waypoint \\
\hline Maximum node speed (m/s) & 10,30 \\
\hline Pause time (seconds) & $30,300,600$ \\
\hline
\end{tabular}

Table 1. Simulation Parameters 
Table 1 shows the simulation parameters. The goal of the simulation experiment is to measure the network lifetime and energy efficiency of wireless routing protocol to react to network topology changes. To measure the efficiency of the network, we applied a variety of node movement and data traffic patterns.

\subsubsection{Simulation space}

Our protocol evaluations and comparisons are based on the simulation of 50 mobile nodes with their random positions over a rectangular $(1500 \mathrm{~m} * 300 \mathrm{~m})$ flat area. We choose a rectangular space in order to force the use of longer routes between nodes than would occur in a square space with equal node density [3]. The simulation environment is in the sparse network. The reason we choose the sparse network is according to [18], in which the balance of the nodal power is critical for extending the network lifetime. In this study, we use a fixed transmission range of 250 meters, which is supported by most of current network cards.

\subsubsection{Movement scenarios}

In the simulation, we use the random waypoint mobility model to generate node movement scenarios. Two important parameters are introduced in this model, pause time and maximum node speed. In NS2, we can use setdest that applies the random waypoint model to generate random mobility. At the start of simulation, each mobile node stays in its initial position on the rectangular topography for a fixed period, which is called pause time and then starts moving independently towards a random 
destination at some random speed, which is uniformly distributed between zero and the specified maximum speed. After the node reaches the target point, it remains stationary for a certain time as specified by the pause time parameter before beginning another movement with random direction and speed. The speed doesn't change when the nodes are mid-way to the destination. The behavior repeats throughout the simulation run time.

Pause time and maximum node speed can determine dynamic topology changes in ad hoc networks. A low pause time and a high maximum speed result in a high number of topology changes while an ad hoc network with high a pause time and a low maximum speed has a low number of topology changes.

In the simulation, we use the setdest utility to generate movement scenarios. Our simulations use movement patterns to generate different pause times: 30 seconds, 300 seconds, and 600 seconds. We do the simulation with two different maximum speeds of node movement, 30 meters per second and 10 meters per second.

\subsubsection{Traffic Pattern}

In the simulation, we use $14 \mathrm{CBR}$ sources and 20 connection nodes with a sending rate of four packets per second. In the traffic pattern, we set each packet with different size, from 64 bytes per packet to 1024 bytes per packet. 


\subsubsection{Simulation Time}

When we simulate DSR and EPDSR, all the connections among the nodes are built at times uniformly distributed between zero and 200 seconds. Then the simulation time should be greater than 200 seconds. Since we configure one movement pattern with pause time 600 seconds, the simulation time should be greater than 600 seconds. We select 1000 seconds as the network running time.

\subsubsection{Energy Model}

During the transmission of the packets in the simulated ad hoc network, the cost used in each operation can be defined in two cases.

1) The transmit power is varied dynamically as the function of the distance between the sender and the receiver.

Stojmenovic and Lin [38] define this metric using a combination of both nodes' lifetime and distance based power metrics. The authors assume that the energy consumption for transmission and reception is a linear function of $d^{\alpha}$.

$$
E(\text { consumed energy })=m * d^{\alpha},
$$

Where $d$ is the distance between two neighbouring nodes and $\alpha$ is a parameter related to the physical environment.

The authors use GPS devices to get the node position information in the transmission. By using the position information, the node transmits packets with minimum required energy. In this algorithm, the relative distance between nodes is 
available to all nodes. In the real network, we could not easily get it. Hence, we need to use GPS to get the distance $d$, and the power consumption of the GPS device is additional. Then we avoid using this energy consumption function and choose another one in which the transmission energy is fixed for packets with same size.

2) The transmission energy is fixed for the same packet size.

Feeney and Nilsson [12] present a linear equation describing the energy consumed by the network interface when a node sends, receives or discards a packet. During all the operations, the energy consumption model is based on the formula:

$$
E(\text { consumed energy })=m * \text { packetSize }+b
$$

Where $m$ and $b$ are empirical constants that are based on the operation, such as send, receive, send broadcast, and receive broadcast. Coefficient $m$ denotes the packet size-dependent energy consumption, and coefficient $b$ is fixed cost that accounts for acquiring the channel and for MAC layer control negotiation. The simulation results presented in [12] confirm the correctness of the linear model in equation (2).

\begin{tabular}{|l|l|l|}
\hline & $m$ (uJoule/byte) & $b$ (uJoule) \\
\hline point-to-point send & 1.9 & 454 \\
\hline point-to-point receive & 0.5 & 356 \\
\hline broadcast send & 1.9 & 266 \\
\hline broadcast receive & 0.5 & 56 \\
\hline promiscuous receive & 0.39 & 140 \\
\hline discard & -061 & 70 \\
\hline
\end{tabular}

Table 2. Energy model parameters

Table 2 shows that sending point-to-point and broadcasting traffic have the same incremental cost, but point-to-point traffic has a higher fixed cost associated with 
the IEEE 802.11 control protocol [12]. In this protocol, point-to-point traffic uses collision avoidance techniques to control collision. Before sending point-to-point packets, the source node broadcasts a request-to-send (RTS) control packet to the destination. The destination node replies a clear-to-send (CTS) packet. This procedure spends energy power. Table 2 also shows that receiving point-to-point traffic has a high fixed cost than receiving broadcast traffic. The reason is that pointto-point traffic spends power on sending two control messages.

Equation (1) is similar to equation (2), as both are linear. By formula (2), the energy consumed in the transmission, receiving, and discarding can be described by a family of linear equation. For each operation, the energy cost has an incremental component that is proportional to the packet size and a fixed coefficient that reflects channel physics characteristics. We use formula (2) in the simulation. Then route selections depend on the packet size. To simplify the simulation, we did not consider the energy consumption in the idle state. For different operations, we illustrate parameters in Table 2, which is taken from [12]. 


\subsubsection{Performance metrics}

\section{Network lifetime}

Network lifetime can be defined in many ways: 1) the time for $\mathrm{P} \%$ of nodes to die; 2) the time for the first node to die; 3) the time for all the nodes to die. The first method is used in data analysis, in which we compare the dead node number.

Nodal Energy difference (Energy consumption difference)

Energy consumption difference between the maximum utilized node and the minimum utilized node is one of the measurements in the analysis. The maximum utilized node is the node with the lowest energy at the end of the simulation. The minimum utilized node is the one with highest energy at the end of the simulation. This metric measures the balance information of energy level among the nodes.

\subsubsection{Simulation Result and Analysis}

\subsubsection{Network lifetime (Number of dead nodes)}

Figure 11 shows the number of dead node as the function with simulation time and pause time in six scenarios. From the observation, whenever the node speed is fixed to $10 \mathrm{~m} / \mathrm{s}$ or $30 \mathrm{~m} / \mathrm{s}$, the results present us two facts: 1) The time for the first node to die in networks with low pause time is later than that in networks with high pause time. 2) The number of dead node is less in low pause time networks than that in high pause time ones. The explanation is that with low pause time, source nodes will change the moving direction frequently. Then the previous path may not useful for the new destination. By issuing route discovery processes, source nodes may get 
new routes with new nodes. Frequently changing routes may lead to balance node energy usage through the whole mobile network. However, with high pause time such as 600 seconds in this simulation, a valid route is always used to forward messages for a long time. This results in quick energy depletion for those frequently used nodes. Continuous use of some routes is one of the disadvantages of DSR. The major goal of new protocol EPDSR is to prevent this.
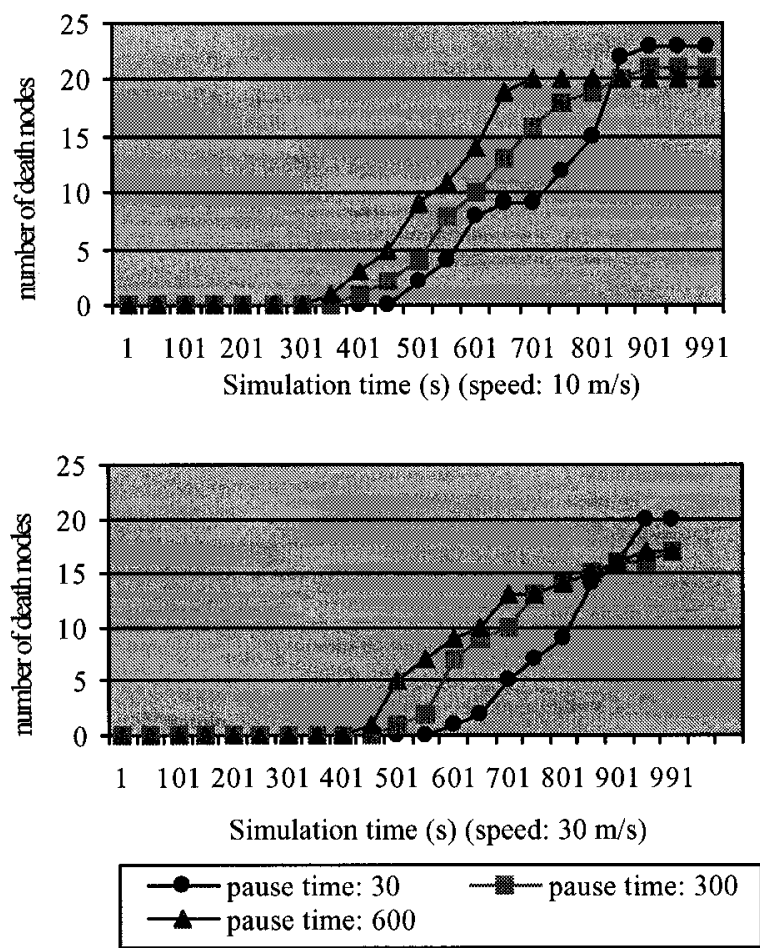

Figure 11. Number of dead nodes as function of pause time

Figure 12 presents the number of dead node as the function with simulation time and node speed in six scenarios. It indicates that when the pause time is fixed to 30 seconds, 300 seconds and 600 seconds, the time of the first node to die will be later in networks with high speed than that in ones with low speed. Figure 12 also shows 
that the number of dead node is less in high speed networks than that in low speed ones. DSR protocol only considers the mobility in the route maintenance. With the high movement rate, there will produce unavailable path due to the node moving out of the transmission range frequently. Then we need more route discovery processes
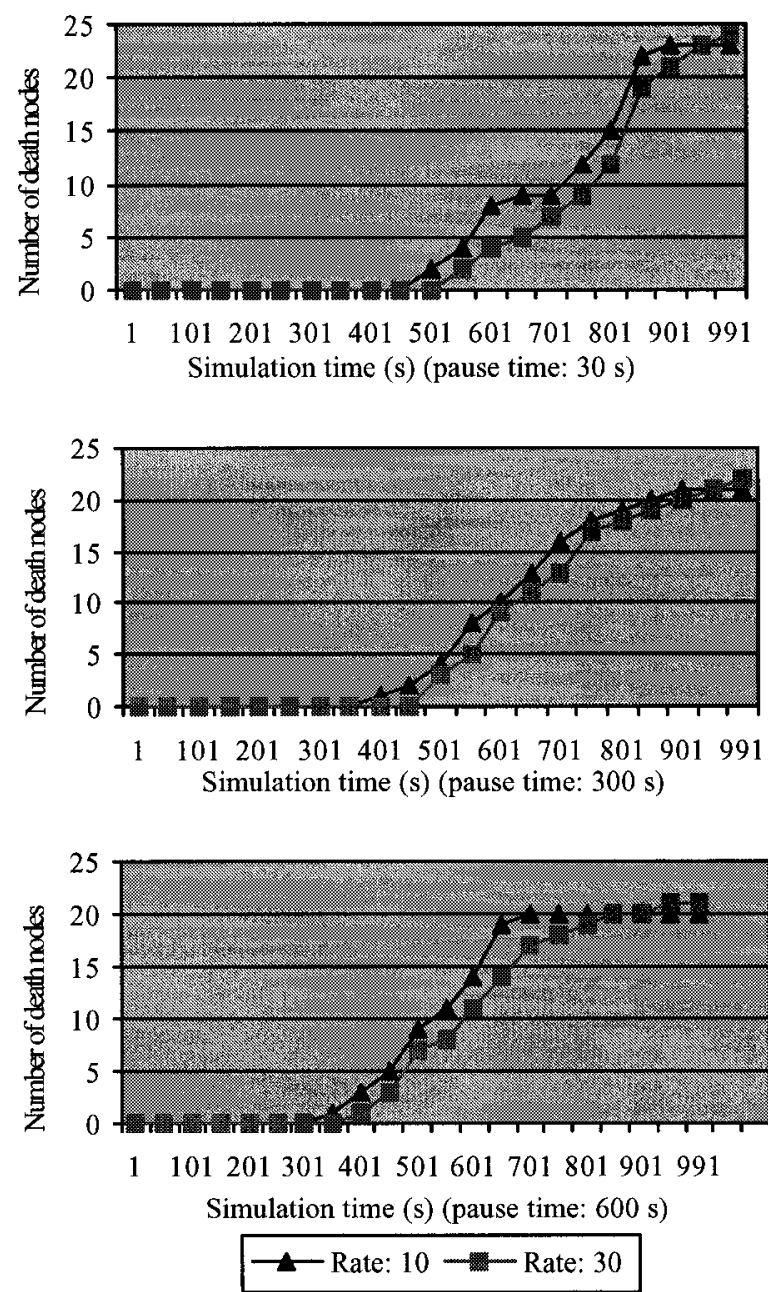

Figure 12. Number of dead nodes as function of node speed to find new paths that contain different nodes. This can prevent overuse of some nodes for a long time and leads to utilize nodes evenly. Then there will be fewer dead nodes in high rate networks than in low rate networks. 
After analysis of Figure 11 and Figure 12, we know that energy efficiency is more critical in the network with low mobility and high pause time. To build a protocol for these stable networks is a challenging job. In the next section, we will introduce a new protocol EPDSR and three performance metrics to compare the performance of DSR and EPDSR, resulting from the average value of six scenarios.

\subsubsection{Difference of nodal energy}

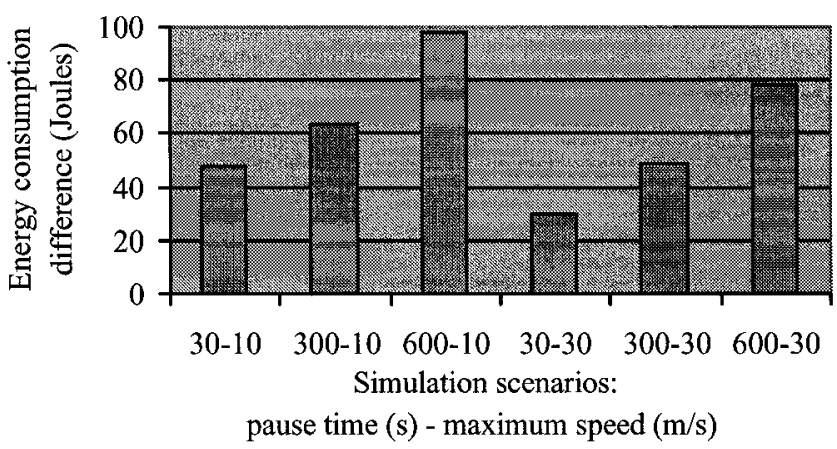

Figure 13. Difference of nodal energy with different scenarios

Figure 13 shows the energy difference between the nodes with the highest and the lowest energy at the end of the simulation in six scenarios. It illustrates that the network will get more energy difference in the stable network with low speed and high pause time. When the speed rate is fixed to $10 \mathrm{~m} / \mathrm{s}$ or $30 \mathrm{~m} / \mathrm{s}$, the network with pause time 600 has the most energy difference. When the pause time is fixed, the network with moving rate $10 \mathrm{~m} / \mathrm{s}$ has less energy difference. It is correct for real networks. In the stable network, the topology always remaining the same and there is no new route to be created for a long simulation time. This will result in some nodes are always on the route and others not. It leads to the increase of energy 
difference among the nodes. In Chapter 5, protocol EPDSR introduces an algorithm to solve this problem, especially in stable networks. 


\section{Chapter 5}

\section{Efficient Power-aware Dynamic Source Routing}

\section{Protocol Simulation}

The main objective of EPDSR is to extend the mobile network lifetime. This is critical in the mobile network since the death of small sets of nodes can lead to the partition of the whole network, which renders the other live nodes unreachable. EPDSR solves this problem by using route discovery algorithm based on cost function of routes. The route selection is controlled by the cost of route and that of each node along the route. EPDSR prevents overuse of small sets of nodes and reduces the energy level variance among nodes in the whole network.

\subsection{Simulation based on DSR}

DSR is a popular protocol and many researches simulate networks based on DSR. We can compare new algorithms with others. There is completed implementation of DSR in network simulator NS2. This makes our simulation easier. Feeney [11] analyzed the energy consumption in three protocols, DSR, AODV and TORA. The results show that DSR generally has better energy utilization distribution between the nodes (based on the maximum and minimum node energy consumption) than other protocols [11]. To improve a wireless protocol with better performance is a challenge but necessary for the communication 
industry. Furthermore, the authors in [11] only consider packets with the same size. In real networks, it is very important to balance the power consumption to transmit messages with different size.

\subsection{Protocol Design}

In the new protocol EPDSR, we add the energy model in the simulation and use the route discovery method based on the cost function. The desirable route has the lowest cost among the possible routes. In DSR, the best route has the shortest hops, without considering the remaining energy of the nodes on the route.

EPDSR maintains multipath between the source and the destination as the result of two-path route discovery. Since when discovering routes, the source issues two RREQs, EPDSR always has more routes than DSR. This can improve the connection of the nodes and avoid frequent disconnections between the source and the destination.

\subsubsection{Multipath route discovery}

The efficiency of DSR depends on the hit radio of route caches. That is, the probability that a route to the destination exists in the cache [42]. Multipath routing in MANET has also received some attention recently. Multipath routing allows establishment of multiple paths between the source and the destination, which provides an easy mechanism to increase the likelihood of reliable data delivery by sending multiple copies of data along different paths. 
Several different multipath routing algorithms have been studied by the prior work. Without considering the energy level of nodes, the author in [42] presented a multipath DSR protocol, in which the simulation results show that two-path DSR protocol increases the connectivity of networks. Napsipuri and Das [29] also present multipath extensions to DSR. In their protocol, the intermediate nodes save multiple routes to the destination. This algorithm reduces the frequency of routing discovery flooding, while maintaining several alternate paths between the source and the destination. Generally, the multipath routing has several characteristics: 1) The frequency of on-demand route discovery is less than that of single path routing. 2) The total control overhead is larger because searching for diverse multiple paths is usually more costly than searching for a single path. 3) Multipath routing can gain some improvement of end-to-end delay in a shared channel MANET. 4) Multipath routing can increase the connectivity of the nodes and reduce packet loss.

DSR also has an option of maintaining multiple routes, so that an alternate route can be used upon failure of the primary one. In DSR, without considering the energy level of the nodes, some nodes perhaps are always on the routes. Continuous use of these nodes can lead to quick depletion of energy. This thesis introduces two-path algorithm by adding energy extension In EPDSR, two kinds of routes have totally different nodes. Alternatively using these routes could balance the node utilization in the network. 


\subsubsection{Cost function and routing algorithm}

We define the energy demand $\boldsymbol{E}_{\boldsymbol{d}}$ for sending packet with size $m$ is: $E_{d}=b^{*} m+c$, where $b$ and $c$ are parameters.

We define the energy supply level $\boldsymbol{E}_{\boldsymbol{s}}$ for node $i$ is:

$E_{s}=E_{i} *\left(E_{i} / E_{0}\right)$, where $E_{i}$ is the current energy of node $i$, $E_{0}$ is the initial energy of node $i$.

We define the cost function $C(r)$ of route $r$ :

$C(r)=\sum C_{i}$, where node $i$ is node in route $r, C_{i}=E_{0} / E_{s}$.

Clearly, the energy supply $E_{s}$ is only part of remaining power. A node with high energy has high energy supply percent. In each transmission, the node uses only parts of the power to participate in the operation, and leaves the remaining parts to participate in the future operations. By this method, the nodes avoid using too much energy at one time. And this can guarantee that the node with more power will be used to transmit the large size packets and the node with less power will be used to transmit the small size packets.

In DSR, when the source node sends packets to the destination, the route selection is based on the shortest hop algorithm. In contrast, EPDSR compares remaining power of each node along the route and the route cost, and then selects the suitable route. In DSR, only mobility can cause the path invalid. In EPDSR, the energy depletion of the node as well as the mobility of the nodes could lead to the invalidation of the selected route. In the following section, we will describe the 
details in route discovery and route maintenance in EPDSR, and compare simulation results of different scenarios.

\subsubsection{Route discovery}

In ad hoc networks, to set up a route, we use route discovery. To make routes ready to use, we need route maintenance. In this section, we discuss the difference between DSR and EPDSR on route discovery and maintenance, and analyze the simulation results of the two protocols.

EPDSR selects the route just as DSR by flooding requests to the neighbouring nodes. Each node in the network stores the received RREQ information such as the source address, destination address, and request ID, etc.

When a source node $\mathrm{S}$ needs to send data packets to a destination node $\mathrm{D}$, it first checks the path cache. If there is no route from the source node to the destination node, the source node issues two RREQs, RREQ1 and RREQ2, and floods them to all its neighbours within the transmission range. In order to detect duplicate RREQ received, each node in the network maintains a list of (identity_number, the source address, destination address, request id, mini_cost). In addition to the address of the source node and destination of the request, source_address and destination_address, the RREQ contains identity_number, path_cost $C(r)$, energy demand $E_{d}$,route_record and request_id. The identity_number is used to distinguish two RREQs. We set 0 and 1 as identity_number for two RREQs. The route_record is an accumulated record of the sequence of hops taken by the route request packet 
as it broadcasts through the ad hoc networking during this route discovery. The unique request_id is a sequence number set by the source node.

The most important difference between the two RREQs (RREQ1 and RREQ2) is energy demand $E_{d l}$ and $E_{d 2}$. The source node can get two kinds of paths by sending these two RREQs. For any node in the network, if it has received one of the RREQs and appended itself on the route, when another RREQ arrives, the node will discard it. By this way, these two kinds of routes have different nodes. In the following description of the route discovery procedure, we use $E_{d}$ to represent $E_{d l}$ and $E_{d 2}$ and use RREQ to represent RREQ1 and RREQ2. We let $E_{d l}$ less than $E_{d 2}$ and

$$
E_{d 2}=\boldsymbol{\beta} E_{d 1} \text {, where } \boldsymbol{\beta} \text { is integer. }
$$

We define $\boldsymbol{\beta}$ as demand difference threshold. We consider the route as major path if it is discovered by issuing RREQ with the energy demand $E_{d I}$.

When RREQ is flooding through the networks, intermediate nodes and the destination node may receive it, and these nodes will decide to forward or discard this RREQ packet.

\subsubsection{Intermediate nodes receive RREQ}

Figure 14 shows the flowchart in this situation.

Case 1: Node $i$ is not the destination, but it has a path to the target in the route cache. 
This intermediate node checks the energy level of each node on this path. If the energy supply $E s$ of each node is greater than the energy demand $E_{d}$ to send packets, this node appends this path on the route and sends back RREP to the source. Otherwise, this node follows the procedure in Case 2.

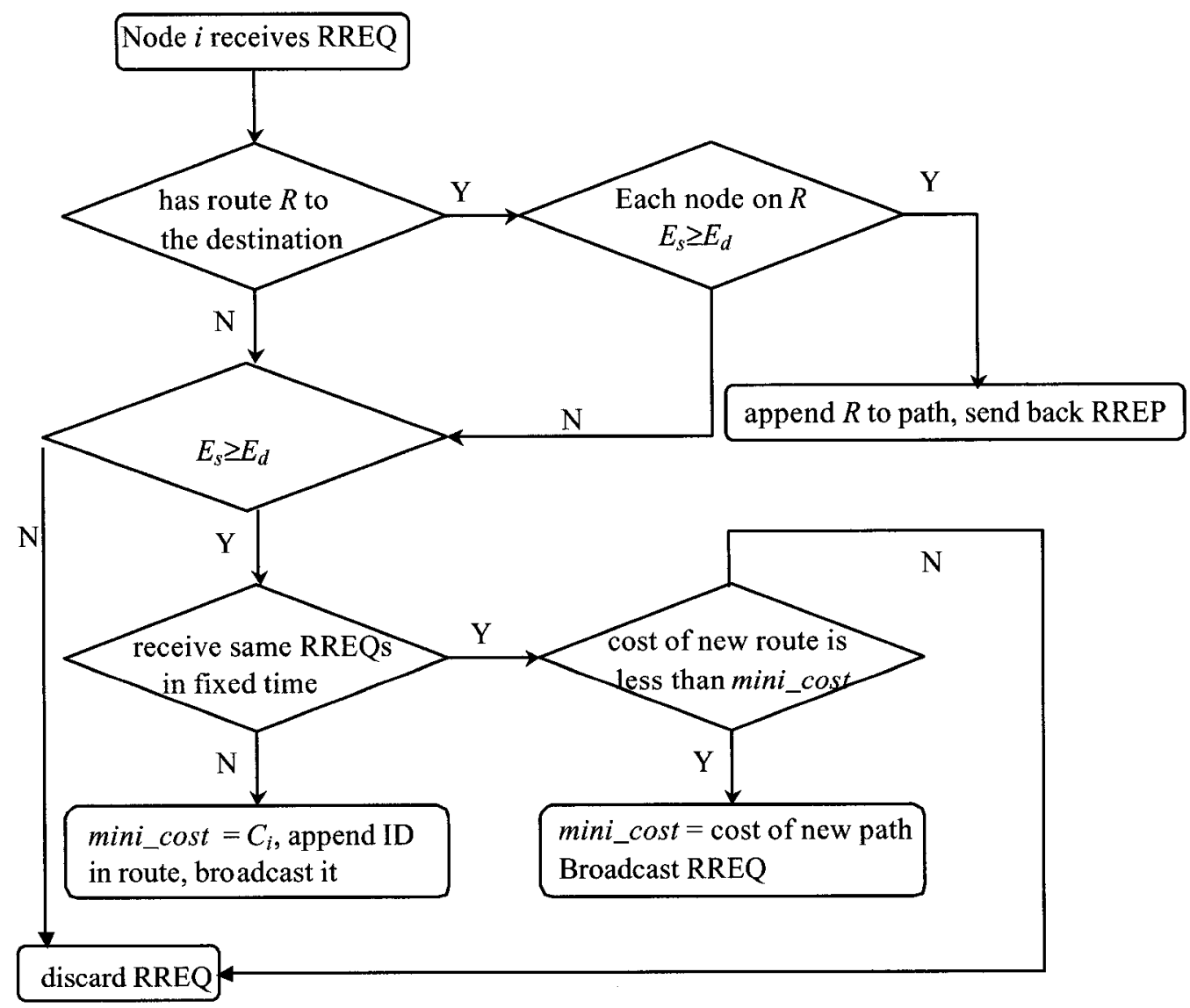

Figure 14. Flowchart when intermediate nodes receive RREQ

Case 2: Node $i$ is not the target and it has no path to the target in the route cache.

The node compares energy demand $\boldsymbol{E}_{\boldsymbol{d}}$ and energy supply $\boldsymbol{E}_{\boldsymbol{s}}$ to decide whether it will broadcast this packet or not. 
1) If energy demand $\boldsymbol{E}_{\boldsymbol{d}}$ is greater than energy supply $\boldsymbol{E}_{\boldsymbol{s}}$, that is $\boldsymbol{E}_{\boldsymbol{s}}<\boldsymbol{E}_{\boldsymbol{d}}$, node $i$ discards the request packet.

2) If energy demand $\boldsymbol{E}_{\boldsymbol{d}}$ is less than or equal to energy supply $\boldsymbol{E}_{\boldsymbol{s}}$, that is $\boldsymbol{E}_{\boldsymbol{s}} \geq \boldsymbol{E}_{\boldsymbol{d}}$, node $i$ will do the following procedures:

i) Node $i$ compares this RREQ with the others received in fixed time by request_id, indentity_number, source_address, desination_address, mini_cost.

a) If there is no such RREQ, node $i$ adds this request packet information into this node, and adds $C_{i}$ to the path_cost, appends this node ID in the route_record, and then broadcasts RREQ with new path_cost to the neighbouring nodes. We keep the route cost in this RREQ packet as mini_cost.

b) If there is the same RREQ in the fixed time, after node $i$ adds $C_{i}$ to path_cost, node $i$ compares the path_cost of the new RREQ with mini_cost. If this RREQ has less path_cost than mini_cost, node $i$ appends its ID in the route_record, records path_cost as mini_cost, and broadcasts it to the neighbours. Otherwise, node $i$ will discard the new RREQ.

\subsubsection{Destination node receives RREQ}

After the first RREQ reaches the destination, the destination waits for a fixed time $T$. Then it compares the path_cost of each route. When time $T$ expires, the destination node selects the path with minimum path cost and sends reply packet 
RREP to the source. After receiving the selected route in RREP, the source puts this route in the route cache.

By issuing two route request packets RREQs, the source node can discover two kinds of routes to the destination. Two request packets are identified by the identity_number 0 and 1 . When the source needs to send packets to the destination, the route with identity_number 0 and energy demand $E_{d l}$ has high priority to be chosen and is called primary route. The route with indentity_number is called secondary route.

\subsubsection{Route Maintenance}

In mobile ad hoc networks, a route may suddenly become broken because only one node roams away. Route maintenance is a critical step in wireless routing protocol and it addresses the problem when a route becomes worse or even broken. When forwarding or sending a data packet to the destination, route maintenance is need to detect if the network topology has changed such that the selected route used by this packet has broken. For mobile protocols with energy model, there exist two reasons for route maintenance: node mobility and energy depletion.

In the first case, the connections between some nodes along transmission path are lost due to the nodes' movement. In this thesis, we still use DSR maintenance algorithm, which is mentioned in Chapter 4, to solve this kind of problem.

In the second case, two possible methods can be applied to deal with energy depletion problem in maintenance. 
1) Global maintenance:

The source node periodically checks the remaining power of all the nodes along the route. When one or more nodes have no enough power for transmission or path cost increases by a fixed value, the source node purges corresponding entry of this path in its route cache. It is very costly for the source node's periodical polls that activate networking flooding. Also this method generates a large mount of extra traffic. This will result in a very high overhead. In building energy efficient routing protocol, we should avoid this maintenance algorithm.

2) Local maintenance:

Each node on the path always monitors the remaining energy after route built. If the remaining energy of any node in the path cannot satisfy the energy limitation, this path will be invalid. Then this node will send an error packet to the source node. This route error message forces the source to initiate route discovery again. This procedure is dependent on the remaining energy capacity of current nodes and then it is a local route maintenance algorithm.

In EPDSR, we use local maintenance method since it can minimize traffic load. In previous energy efficient protocols, authors only consider route link cost. Our route maintenance method pays more attention to individual nodal energy. When originating or forwarding a packet using a cached route, each node transmitting the packet is responsible for confirming that the packet has been received by the next hop along the route. If the node did not receive the confirmation, it will retransmit 
for maximum number of times. If it still could not receive the confirmation packet, it will send back to the source node a Route Error message. Then the source issues another route discovery process. In DSR, the only reason to produce Route Error message is that one of the nodes ran out of the transmission range.

In EPDSR, before a route is used to transmit packets, the energy level of each node on this route should be checked. A route will be deleted when the cost of any node on the route meets the following condition: $C_{i}(t)-C_{i}\left(t_{0}\right) \geq \gamma$, where $t$ is the current time, and $t_{0}$ is route discovery time. We define $\boldsymbol{\gamma}$ as cost function threshold.

\subsubsection{Difference between EPDSR and DSR}

EPDSR is simulated based on DSR The following table shows the difference between them.

\begin{tabular}{|c|l|l|}
\hline & DSR & EPDSR \\
\hline Route discovery & & \\
\hline RREQ number & 1 & 2 \\
\hline RREQ flooding & $\begin{array}{l}\text { Each node floods the first } \\
\text { RREQ to each neighbour in } \\
\text { transmission range. }\end{array}$ & $\begin{array}{l}\text { Only the node with enough power } \\
\text { supply on the route and with lowest } \\
\text { cost floods the RREQ. }\end{array}$ \\
\hline Route selection & Shortest hops & Shortest cost \\
\hline Route maintenance & Mobility of nodes & $\begin{array}{l}\text { Mobility of nodes } \\
\text { Depletion of energy }\end{array}$ \\
\hline
\end{tabular}

Table 3. Difference between EPDSR and DSR 


\subsubsection{Difference between DSR, MTPR, MBCR, and MMBCR}

Table 4 shows the difference of route discovery between EPDSR and the previous power-aware protocols. EPDSR concentrates on the remaining power of

\begin{tabular}{|l|l|l|l|}
\hline & Least total energy & Avoid overusing some nodes & Avoid lowest energy nodes \\
\hline EPDSR & & V & V \\
\hline MTPR & V & & \\
\hline MBCR & & $\sqrt{ }$ & \\
\hline MMBCR & & & $\sqrt{ }$ \\
\hline
\end{tabular}

Table 4. Difference between EPDSR and other power-aware protocols

each node and selects the routes based on the principle of energy balance among nodes. Up to now, it is the better way to extend the network lifetime. In [4], the author simulated MTPR, MBCR, and MMBCR. In route maintenance, the author did not consider energy usage and depletion in simulation. However, we consider the energy level as one of two major factors in the maintenance in EPDSR. 


\subsection{Simulation result and performance analysis}

EPDSR simulation is based on the same environment for DSR simulation in NS2. The mobility is the key character of mobile networks and density is a major factor for the connection of mobile networks. We evaluate how node mobility can affect protocol performance. We used pause time of 30,300, and 600 seconds and the maximum speed of nodes is selected to $10 \mathrm{~m} / \mathrm{s}$ and $30 \mathrm{~m} / \mathrm{s}$. Then we design several scenarios for comparing the performance of EPDSR and DSR based on different mobility patterns and density patterns.

\subsubsection{Analysis of two threshold $\beta$ and $\gamma$}

In the route maintenance of protocol EPDSR, because of the dynamic character of path cost, the selected route cannot work for a very long period. The reason is that if any route remains for a long time, some of nodes may exhaust energy quickly. Accumulated depleted nodes may lead to the partition of the network. When a route is discovered, it will be put in the cache, and can be accessed when it is needed. In EPDSR, energy depletion and node mobility can invalidate cache entries. Cache invalidation is very costly since route rediscovery is done by flooding packets throughout the whole network.

In EPDSR, we use demand difference threshold $\beta$ and cost threshold $\gamma$ to determine the frequency of route invalidation. When we decide the value of $\beta$ and $\gamma$, route discovery number should be considered since more discovery processes waste 
more energy. Suitable threshold $\beta$ and $\gamma$ are very important factors for energy saving.

\subsubsection{Analysis of demand difference threshold $\beta$}

In this experiment, we use four scenarios (scenario $30-10,30-30,300-10,300-$ 30) respectively with four threshold values $0,5,10,20$. For each scenario, five patterns are created to simulate and average results are used to analyze the performance. In order to compare results, we set the fixed cost threshold $\gamma$ to 2 . We analyze the route discovery numbers in each scenario.

Figure 15 presents the number of route discovery in each scenario with different threshold $\beta$. It illustrates that when $\beta$ is equal to 5 , we get the lowest route discovery number in each scenario. The lower or higher threshold value leads to higher route discovery number. When $\beta$ is very small or almost equal to 0 , if the primary routes

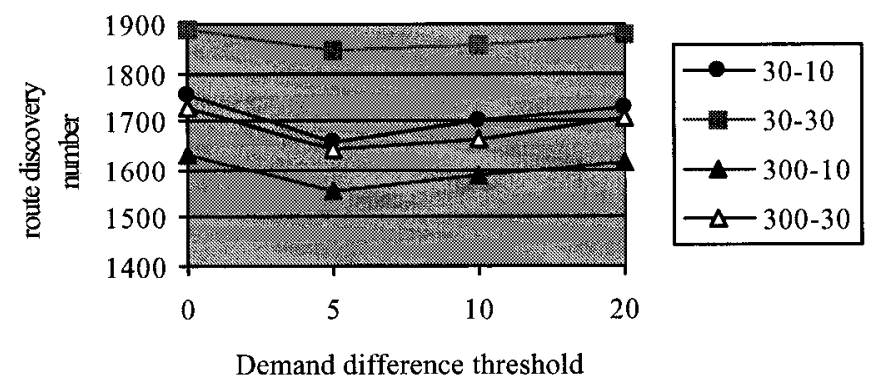

Figure 15. Route discovery number with different demand difference thresholds 
get invalidation error, the secondary routes may only be available for a relatively short time. This is because some nodes' energy in these routes may have a little more energy than those in the primary routes. When we use the primary route to transmit some message, some nodes in the secondary routes may be in another route and are used for other transmissions. After the primary routes become invalid, the source uses a secondary route to transmit packets. This route may be maintained for a short time since some nodes with low energy are on it. The cost change of these nodes may lead to another invalidation error message. When $\beta$ is very large, if nodes do not contain high power, we cannot get secondary routes through this route discovery method. In the later simulation, demand difference threshold $\beta$ is set to 5 .

\subsubsection{Analysis of cost function threshold $\boldsymbol{\gamma}$}

In this experiment, we use same scenarios as the last experiment. We set demand difference threshold $\beta$ to 5 . Cost function threshold $\gamma$ is set to $0.2,2$, and 20 .

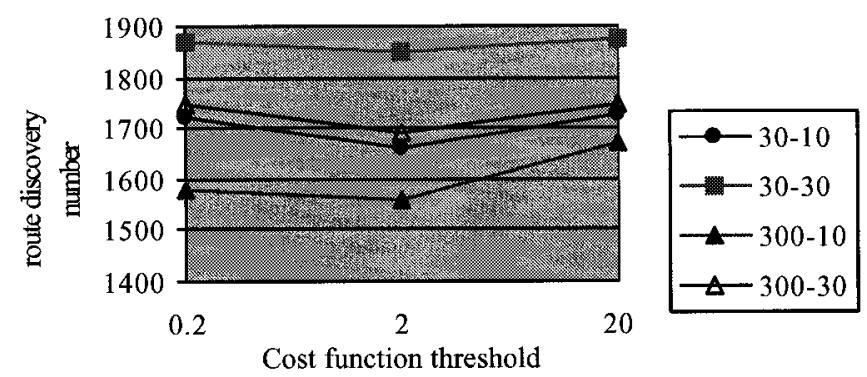

Figure 16. Route discovery number with different cost function thresholds 
Figure 16 presents the number of route discovery in each scenario with different threshold $\gamma$. It shows that when $\gamma$ is equal to 2 , there exists relatively low route discovery number. If threshold $\gamma$ is very high, route invalidation errors will be created rarely. Routes always keep working for a long time and this might overuse some nodes in these paths. If threshold $\gamma$ is very slow, we will get very high cache invalidation rate, and might lead to unnecessary route request packet flooding in the network. This threshold affects the performance of protocol EPDSR. In the later simulation, we set cost function threshold $\gamma$ to 2 .

\subsubsection{Performance Metrics}

\section{Network lifetime}

Network lifetime can be defined in many ways: 1) the time for P\% of nodes to die; 2) the time for the first node to die; 3 ) the time for all the nodes to die. We use the first method in the analysis, in which we compare the dead node number within the simulation time.

\section{Nodal Energy difference (Energy consumption difference)}

Energy consumption difference between the maximum utilized node and the minimum utilized node is one of the measurements in the analysis. The maximum utilized node is the node with the lowest energy at the end of the simulation. The minimum utilized node is the one with highest energy at the end of the simulation. This metric measures the balance information of energy level among the nodes. 
Energy consumption per byte packet

Energy consumption per byte packet is the ratio between the total communication cost in the network and the total number of packet bytes received by their final destination. By using this metric, the protocol can show how the packets have been successfully received through limit power.

\subsubsection{Performance Comparison when Varying Mobility}

Node mobility is a key reason in route selection and maintenance in ad hoc networks. In this section, we analyze protocol performance under different mobility and traffic patterns. Two factors are presented for designing mobility pattern, pause time and maximum speed of node movement. First, we set maximum speed respectively to fixed value $10 \mathrm{~m} / \mathrm{s}$ and $30 \mathrm{~m} / \mathrm{s}$ and choose pause time as 30 seconds, 300 seconds, and 600 seconds.

\subsubsection{Network lifetime (dead node number)}

Figure 17 and Figure 18 show the dead node number in EPDSR and DSR under different maximum speed and pause time. 

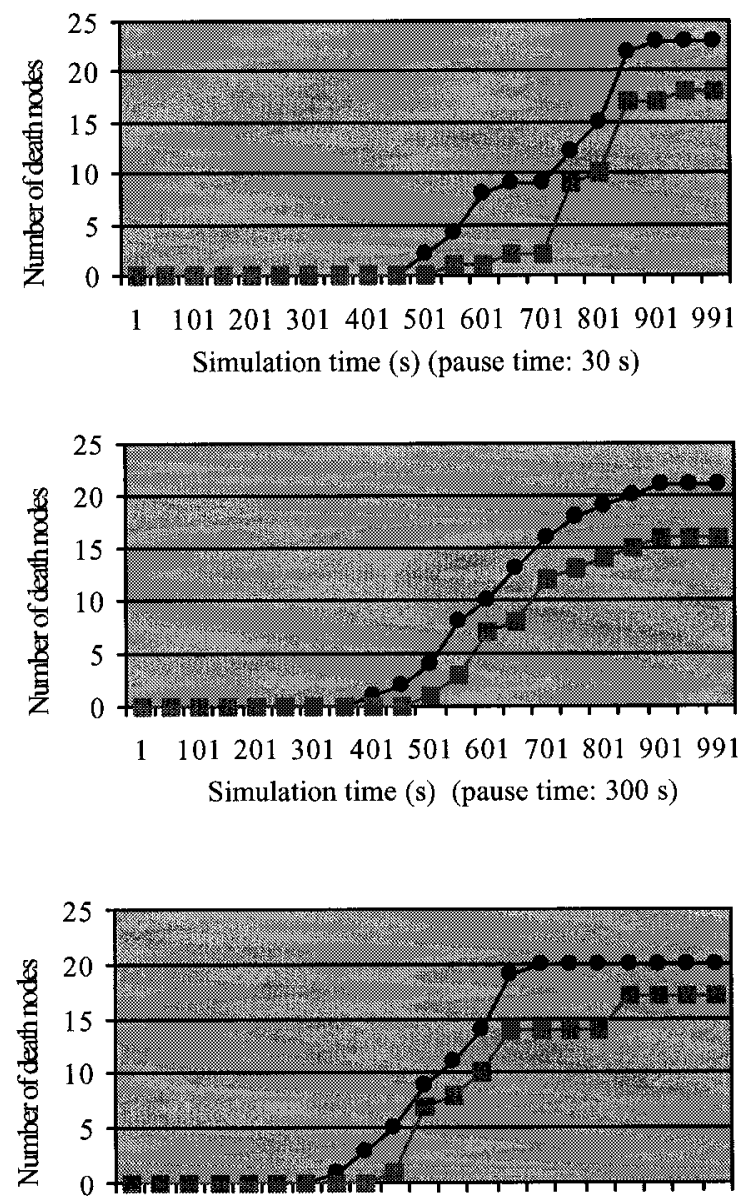

1101201301401501601701801901991 Simulatin time (s) (pause time: $600 \mathrm{~s}$ ) $\rightarrow-$ DSR $\rightarrow-$ EPDSR

Figure 17. Comparison of dead node number (node speed: $10 \mathrm{~m} / \mathrm{s}$ ) 

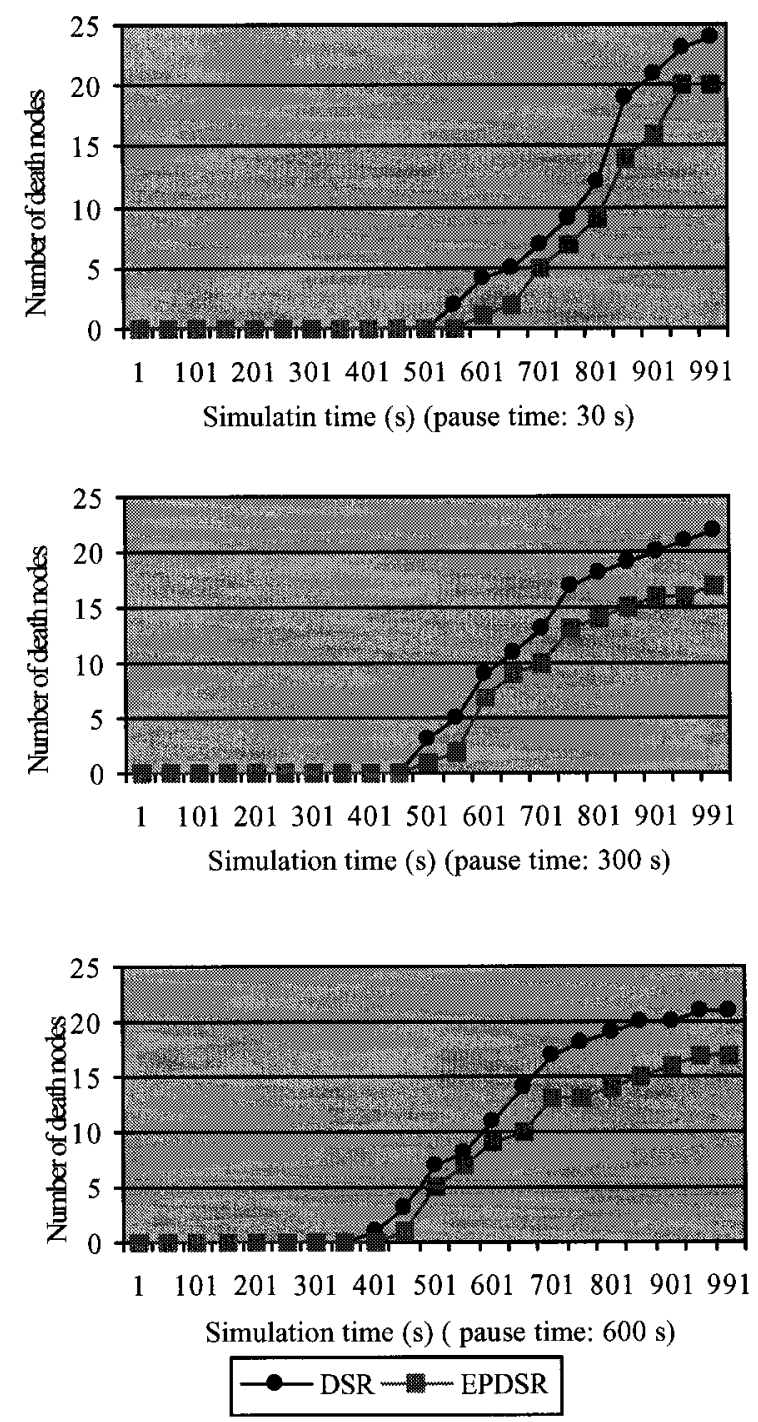

Figure 18. Comparison of dead node number (node speed: $30 \mathrm{~m} / \mathrm{s}$ )

We can observe three features of this metric in the simulation of EPDSR and DSR.

First, the number of dead node in EPDSR is parallel to that in DSR, which means that the implementation of the EPDSR is correct. Secondly, for all scenarios, EPDSR has less dead node number than DSR. The dead node number is reduced by more than $20 \%$. It is as good as we expected. Finally, EPDSR results in more 
decrease of dead node number in stable networks with low moving rate and high pause time. For the network with speed $10 \mathrm{~m} / \mathrm{s}$, the number of dead node is reduced by around $22 \%$. For network with speed $30 \mathrm{~m} / \mathrm{s}$, the number of dead node is reduced by about $21 \%$. The result demonstrates that EPDSR has better performance in the network with slightly low speed.

\subsubsection{Nodal Energy difference}

Figure 19 presents energy difference between nodes with the highest power and the lowest power at the end of running time in DSR and EPDSR. It shows that in all simulation scenarios, power difference is reduced about $40 \%$ in EPDSR. It means that node utilization in EPDSR is generally more balanced in EPDSR than that in DSR. This figure also illustrates that EPDSR produces more energy difference decrease in stable networks with high pause time or low node speed. In scenarios with node maximum speed $10 \mathrm{~m} / \mathrm{s}$, the energy different decrease is about $37 \%$ $42 \%$. This decrease is about $30 \% \sim 40 \%$ in scenarios with node speed $30 \mathrm{~m} / \mathrm{s}$. The main reason is that EPDSR introduces more route maintenance processes in stable networks. In the network with relatively low mobility and high pause time, some sets of nodes are always used for a long time, this will lead to the nodal cost change exceeds threshold $\gamma$, and results in the creation of route error in route maintenance, which leads to new route discovery process. A new route always prevents selecting some old nodes in previous route because of their low energy level and high path 
cost level. The more different paths are created, the more balance usage of the nodes.
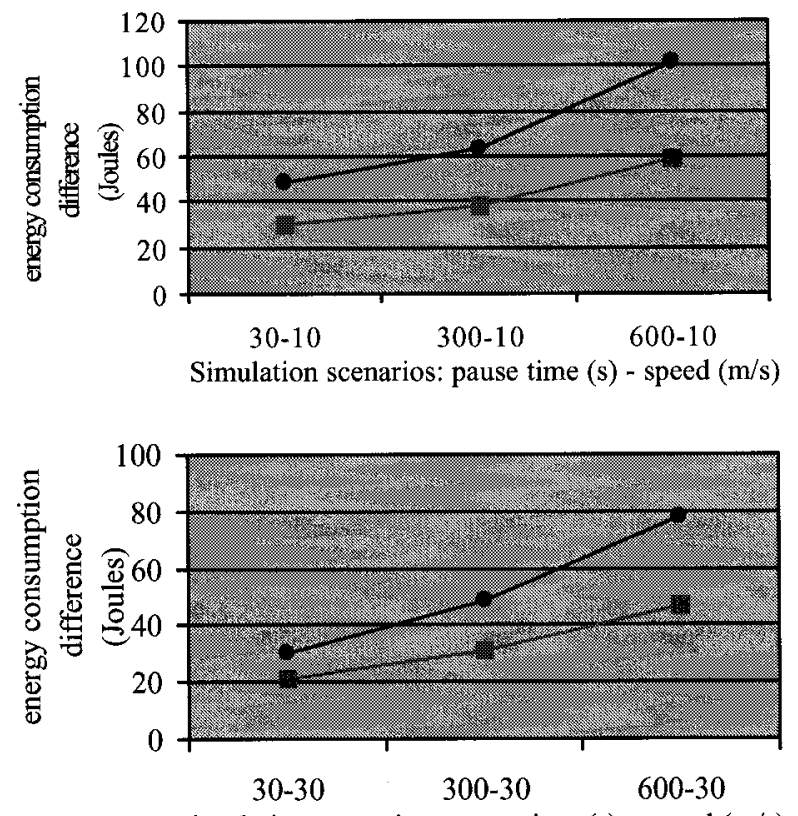

simulation scenarios: pause time (s) - speed $(\mathrm{m} / \mathrm{s})$

$$
\rightarrow-\text { DSR }- \text { iv- EPDSR }
$$

Figure 19. Nodal energy difference in DSR and EPDSR

\subsubsection{Energy consumption per byte packet}

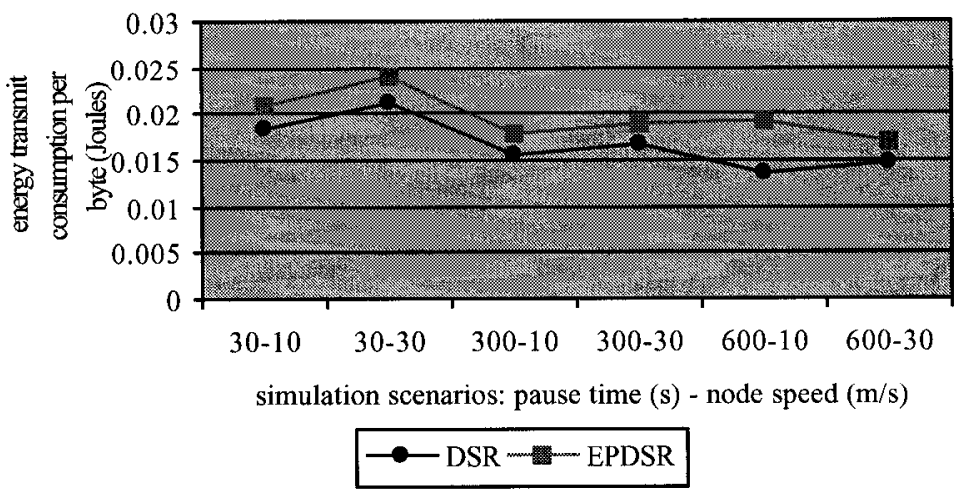

Figure 20. Energy consumption per byte packet 
Figure 20 presents the energy consumption per byte packet in DSR and EPDSR. It shows that in all scenarios, EPDSR has about $14 \%$ more energy consumption for each byte packet transmission than DSR. This feature can be explained by two reasons. First, EPDSR maintenance procedure considers not only node mobility but also the cost level of each node, thus it issues more route error messages and more route rediscovery processes. Route discovery is very costly. The second reason is obviously that EPDSR applies the shortest cost algorithm to locate routes, but DSR uses shortest hops to discover routes. EPDSR always prefers the route with more hops and with the lowest cost routes to those with the shortest hops in DSR. In both protocols, each node consumes same power in the transmission for fixed size packets according to equation (2) in Chapter 4. Figure 20 also shows that EPDSR produces more energy consumption increase in stable networks. In networks with node speed $30 \mathrm{~m} / \mathrm{s}$, the energy consumption increase is about $12 \% \sim 14 \%$. However, in networks with node speed $10 \mathrm{~m} / \mathrm{s}$, this increase is about $14 \% \sim 15 \%$. The reason is that stable networks with EPDSR produce more discovery processes that consume more power.

Although protocol EPDSR may consume more power in the transmission, it has many merits. We will introduce a measuring yardstick to analyze the general performance of EPDSR and DSR at the end of this chapter. 


\subsubsection{Summary of EPDSR performance analysis}

Here, we use six scenarios to get average performance result. Pause time is respectively set to 30 seconds, 300 seconds and 600 seconds, and node maximum speed is set to $10 \mathrm{~m} / \mathrm{s}$ and $30 \mathrm{~m} / \mathrm{s}$. For each scenario, we create five different traffic and movement patterns. We simulate these five patterns for every scenario in EPDSR and compare average performance with that in DSR in three aspects, dead node number, nodal difference and energy consumption in transmission for each byte of packet.

\begin{tabular}{|c|c|c|c|}
\hline \multirow[t]{2}{*}{ Pause time } & \multirow[t]{2}{*}{ Performance Metrics } & $10 \mathrm{~m} / \mathrm{s}$ & $30 \mathrm{~m} / \mathrm{s}$ \\
\hline & & average & average \\
\hline \multirow[t]{3}{*}{30 second } & dead node number decrease & $22.52 \%$ & $21.36 \%$ \\
\hline & nodal energy difference decrease & $37.40 \%$ & $29.34 \%$ \\
\hline & $\begin{array}{l}\text { energy consumption per byte packet } \\
\text { in transmission increase }\end{array}$ & $14.23 \%$ & $12.21 \%$ \\
\hline \multirow[t]{3}{*}{300 second } & dead node number decrease & $23.32 \%$ & $21.98 \%$ \\
\hline & nodal energy difference decrease & $40.47 \%$ & $35.65 \%$ \\
\hline & $\begin{array}{l}\text { energy consumption per byte packet } \\
\text { in transmission increase }\end{array}$ & $14.67 \%$ & $13.34 \%$ \\
\hline \multirow[t]{3}{*}{600 second } & dead node number decrease & $23.95 \%$ & $22.43 \%$ \\
\hline & nodal energy difference decrease & $41.55 \%$ & $39.78 \%$ \\
\hline & $\begin{array}{l}\text { energy consumption per byte packet } \\
\text { in transmission increase }\end{array}$ & $15.20 \%$ & $14.12 \%$ \\
\hline
\end{tabular}

Table 5. Average values of EPDSR performance with varying mobility

The performance of EPDSR and DSR, in terms of different maximum node speed and different pause time is evaluated. The result in Table 5 illustrates that at low mobility rate and high pause time, protocol EPDSR has better performance than 
DSR: fewer dead nodes are produced, and energy consumption becomes more balanced. The drawback of EPDSR is that it introduces more energy consumption for each byte packet in the transmission. Although EPDSR has its own disadvantage, its performance is still better than DSR in network lifetime and network power usage balance. Generally, we get the following conclusion: 1) The number of dead nodes in EPDSR decreases around 22\% compared with that in DSR. 2) Protocol EPDSR greatly reduces energy variance by about $37 \%$, prevents overuse of small sets of nodes, and ultimately extends the network partition time. 3) However, average energy consumption for each byte packet transmission in EPDSR increases approximately by $14 \%$.

\subsubsection{Performance comparison when varying density}

Density is one of the important factors to affect protocol performance. In This experiment, we evaluate the behaviour of EPDSR in sparse networks and dense networks. From Table 5, we get the conclusion that EPDSR has better performance in relatively stable networks. Therefore, in the simulation, we set max speed to 10 $\mathrm{m} / \mathrm{s}$ and set pause time to 30 seconds, 300 seconds, and 600 seconds. A MANET consisting of 75 mobile nodes with their random positions in the area of $1500 \mathrm{~m} \mathrm{x}$ $300 \mathrm{~m}$ is generated. We still consider total of 14 traffic sources with a sending rate of 4 packets/sec. All the connections start at a random time to emulate real network environment. For each scenario, we create five different traffic and movement 
patterns and get average results. We compare the results of these simulations with those we mentioned in 5.3.3.

From Table 6, we can summarize the following points. Comparing the simulation in sparse networks, EPDSR has less dead node number decrease and less energy difference decrease in dense networks. We can get the conclusion that EPDSR has not improved network balance greatly in dense networks. Thus EPDSR has better performance in sparse networks than that in dense ones. However, the energy consumption increase of each byte packet in dense networks is not so high as it in sparse ones.

\begin{tabular}{|l|l|r|r|}
\hline & Performance Metrics & Sparse & Dense \\
\cline { 3 - 4 } & & average & average \\
\hline \multirow{2}{*}{$\begin{array}{l}\text { j0 second } \\
\text { pause time }\end{array}$} & dead node number decrease & $22.52 \%$ & $13.58 \%$ \\
\cline { 2 - 4 } & nodal energy difference decrease & $37.40 \%$ & $16.25 \%$ \\
\cline { 2 - 4 } & $\begin{array}{l}\text { energy consumption per byte packet } \\
\text { in transmission increase }\end{array}$ & $14.23 \%$ & $12.21 \%$ \\
\hline \multirow{3}{*}{$\begin{array}{l}\text { pause time } \\
\text { pause }\end{array}$} & dead node number decrease & $23.32 \%$ & $10.36 \%$ \\
\cline { 2 - 4 } & nodal energy difference decrease & $40.47 \%$ & $21.69 \%$ \\
\cline { 2 - 4 } $\begin{array}{l}\text { energy consumption per byte packet } \\
\text { in transmission increase }\end{array}$ & $14.67 \%$ & $13.23 \%$ \\
\hline $\begin{array}{l}600 \text { second } \\
\text { pause time }\end{array}$ & dead node number decrease & $23.95 \%$ & $14.56 \%$ \\
\hline & nodal energy difference decrease & $41.55 \%$ & $25.21 \%$ \\
\hline & $\begin{array}{l}\text { energy consumption per byte packet } \\
\text { in transmission increase }\end{array}$ & $15.20 \%$ & $15.12 \%$ \\
\hline
\end{tabular}

\section{Table 6. Average values of EPDSR performance with varying density}

In the sparse networks, the role of nodes for avoiding network partitioning is more crucial. The overuse of some nodes is very critical for the whole network 
running. The more route breaks and route rediscoveries can balance the power consumption among nodes. All of these could be realized by cost function discovery algorithm and two-path discovery algorithm in EPDSR. As for dense networks, since there always exist enough nodes in the transmission range, it seems to be more important to reduce overall ene rgy consumption so as to prolong the lifetime of each node. Therefore, in dense networks, we prefer DSR to EPDSR.

\subsubsection{Simulation result evaluation}

Our objective of protocol EPDSR is to develop algorithms that achieve good communication performance subject to constraints in energy consumption. Therefore performance evaluate measures must reflect the characteristics of the routing problem and the energy consumption limitations. [26] introduces two measures, the call blocking probability $P_{b}$ and the average energy per session $E_{s}$. A call global performance yardstick $Y$ is mentioned as the average transmission ratio per energy unit, i.e.

$$
Y=\frac{1-P_{b}}{E_{s}}
$$

Blocking probability is the number of sessions, which are blocked during one interval. Blocking probability reflects the mobility and the stability of the networks. In our evaluation analysis, we use packets drop ratio as blocking probability. $E_{s}$ reflects the energy consumption in operations. Since in our simulation, the packet size of each session is different, we chose average energy consumption for each byte 
packet transmission as $E_{s}$. The goal of the energy efficient protocols is to increase the value of $Y$.

Table 7 presents values of $P_{b}, E_{s}$ and yardstick $Y$ in simulations for different traffic patterns and movement patterns in DSR and EPDSR. In all scenarios, the packets drop rate in EPDSR is less than that in DSR but the power consumption for each byte packet transmission is more than that in DSR. The interesting thing is that by using EPDSR, sparse networks and dense networks get totally different performance in yardstick. In sparse networks, the performance yardstick Y in EPDSR achieves higher value as we expected. However in dense networks, EPDSR gets lower yardstick value.

\begin{tabular}{|l|l|l|l|l|l|l|l|}
\hline \multicolumn{3}{|c|}{} & \multicolumn{3}{l|}{ sparse networks (50 nodes) } & \multicolumn{3}{l|}{ dense networks (75 nodes) } \\
\hline & protocol & $P_{b}$ & $E_{s}$ & $Y$ & $P_{b}$ & $E_{s}$ & $Y$ \\
\hline $30-10$ & DSR & 0.248 & 0.01845 & 40.76 & 0.185 & 0.01658 & 49.16 \\
\cline { 2 - 8 } & EPDSR & 0.138 & 0.02103 & 40.98 & 0.165 & 0.01861 & 44.87 \\
\hline $30-30$ & DSR & 0.201 & 0.02134 & 37.44 & 0.152 & 0.01733 & 48.93 \\
\cline { 2 - 8 } & EPDSR & 0.1 & 0.02395 & 37.6 & 0.136 & 0.01941 & 44.51 \\
\hline $300-10$ & DSR & 0.298 & 0.01565 & 44.86 & 0.219 & 0.01531 & 51.01 \\
\cline { 2 - 8 } & EPDSR & 0.192 & 0.01795 & 45.03 & 0.178 & 0.01734 & 47.38 \\
\hline $300-30$ & DSR & 0.228 & 0.01678 & 46.01 & 0.157 & 0.01574 & 53.56 \\
\cline { 2 - 7 } & EPDSR & 0.12 & 0.01903 & 46.23 & 0.144 & 0.01778 & 48.14 \\
\hline
\end{tabular}

Table 7. Simulation result evaluation in networks

The reason of this phenomenon is that the effect of EPDSR in dense networks is less than that in sparse ones. EPDSR can decrease drop rate greatly in sparse networks, but not in dense networks. Yardstick $Y$ is such an effective measurement for wireless 
networks since it reflects two characteristics of MANET, mobility and energy limitation. The fact that EPDSR gets less value of $Y$ than DSR proves that EPDSR is not suitable for dense networks. 


\section{Chapter 6}

\section{Conclusions and future work}

Reducing power consumption in ad hoc networks has received increased attention among researchers in recent years. Ad hoc wireless networks are power constrained since nodes operate with limited battery energy. Several efficient power-aware routing protocols have been developed for mobile networks. To reduce total transmission power and to prolong the lifetime of each node are two important aspects in MANET. In this thesis, we consider these two characters in the simulation. To maximize the lifetime of these networks, network-related transactions through each mobile node must be controlled such that the power dissipation rates of all nodes are nearly same. In this thesis, a new energy efficient protocol EPDSR is proposed.

We can point out contributions from this thesis:

This thesis provided a study of wireless network routing protocols. Two categories of routing protocols are discussed, table-driven and on-demand routing protocols. We overview the classification of the conserving energy routing protocols. Three different groups are introduced: switching on/off transmitters to save energy, routing based on the metric of energy consumption, 
and network topology control by adaptively adjusting transmitter radios. We concentrated on the routing based on the me tric of energy consumption.

We designed and implemented route discovery in EPDSR based on DSR using network simulator NS2. In EPDSR, we introduced two-path discovery algorithm and cost function route selection algorithm. In the route discovery, the source issued two route request packets, and flooded them to the neighbours. The nodes only respond one of these two RREQ packets. When receiving one request packet, intermediate nodes with enough energy supply forward it. The destination source chose the route according to the cost of each route. The functions of energy supply, energy demand, and the cost of routes are introduced in Chapter 5.

We designed and implemented route maintenance in EPDSR. EPDSR considered the effect of mobility and power depletion of nodes. In node mobility aspect, EPDSR uses the same maintenance method as that in DSR. In order to minimize control traffic, EPDSR adopts local approach maintenance. When the cost change of any node along the route is greater than the fixed threshold, the route will be deleted.

We created several scenarios with different traffic patterns and movement patterns using sedest in NS2. In order to evaluate the behaviour of EPDSR in different networks, we analysed and compared the performance of EPDSR and DSR in different scenarios. We get some conclusions as following in sparse 
networks: 1) The number of dead nodes in EPDSR decreases around $22 \%$ compared with that in DSR. 2) Protocol EPDSR greatly reduces energy variance by about $37 \%$ and prevents overuse of small sets of nodes. 3) However, average energy consumption for each byte packet transmission in EPDSR increases approximately by $14 \%$. 4) EPDSR has better performance in stable networks with low rate and high pause time. At last, we evaluate the performance of EPDSR in dense networks. EPDSR achieves better performance in sparse networks than in dense networks. The detailed analysis is presented in Chapter 5.

Although we have done many works on cost function discovery and maintenance algorithm, there still exists more aspects in the future work.

In order to prove the correctness of our algorithm, we need to implement it in the other protocols such as AODV and TORA.

EPDSR can improve efficiency for sparse networks. But it cannot achieve good performance in dense networks. Under this condition, we prefer DSR to EPDSR. In real networks, there exists some situation that at the beginning of the simulation, networks belong to dense ones. However, it will become sparse after death of many mobile nodes. In order to save energy, we should use DSR at the beginning and use EPDSR after some time. In future work, we need study the condition under which we transfer DSR to EPDSR in the simulation. 
In this thesis, the new protocol EPDSR can extend network lifetime and increase energy level balance among the nodes. However, it still increases energy consumption for transmission. We need more modifications to decrease this metric value such as turning off the idle nodes and reducing any kinds of request packet numbers.

In both EPDSR and DSR, when a node sends RREP to the destination, it floods this packet to all its neighbours. All the neighbouring nodes will spend power on receiving this message. It will waste a large mount of energy. In future work, we should consider the direction and location of nodes. By using a sensor facility, nodes only send request messages to others in the specific area. 


\section{References}

[1] Banerjee, S., Minimum Energy Paths for Reliable Communication in Multihop Wireless networks, International Conference on Mobile Computing and Networking, pages 146-156, 2002.

[2] Brown, T.X. and Zhang, S., Optimal power aware routing in a wireless ad hoc network, Proceedings of IEEE LANMAN Workshop, pages 102-105, 2001.

[3] Broch, J., Maltz, D.A., Johnson, D.B., Hu, Y.-C. and Jetcheva, J., A Performance Comparison of Multi-Hop Wireless Ad Hoc Network Routing Protocols, Proceedings of the 4th Annual ACM/IEEE International Conference on Mobile Computing and Networking (MOBICOM'98), pages 85-97, Dallas, TX, USA, October 1998.

[4] Cano, J.C, Manzoni, P., A Performance Comparison of Energy Consumption for mobile Ad Hoc Networks Routing Protocols, Proceedings of the 8th International Symposium on Modeling, Analysis and Simulation of Computer and Telecommunication Systems, pages 57-64, 2000.

[5] Chang, J.H. and Tassiulas, L., Routing for maximum system lifetime in wireless ad-hoc Networks, Proceedings of 37th Annual Allerton Conf. on communication, Control, and Computing, Monticello, IL, September 1999.

[6] Chang, J., and Tassiulas, L., Energy Conserving Routing in Wireless Ad Hoc Networks, Proceedings of IEEE INFOCOM, Israel, pages 22-31, 2000. 
[7] Chiang, C.-C., Routing in Clustered Multihop Mobile Wireless Networks with Fading Channel, Proceedings of IEEE SICON'97, pages 197-211, April1997.

[8] Chen, B., Jamieson, K., Balakrishnan, H. and Morris, R., Span: An EnergyEfficient Coordination Algorithm Topology Maintenance in Ad Hoc Wireless networks, Wireless Networks, Volume 8, Issue 5, pages 481-497, 2002.

[9] Corson, S. and Park, V., A Highly Adaptive Distributed Routing Algorithm for Mobile Wireless Networks, INFOCOM 1997, volume 3, pages 1405-1413, January 1999.

[10] Doshi, S., Brown, T. X., Minimum Energy Routing Schemes for a Wireless Ad Hoc network, IEEE INFOCOM, 2002.

[11] Feeney, L. M., A Taxonomy for Routing Protocols in Mobile Ad Hoc Networks, SICS technical Report T99:07, ISRN: SICS-T-99/07-SE,1999.

[12] Feeney, L.M. and Nilsson, M., Investigating the Energy Consumption of a Wireless Network Interface in an Ad Hoc Networking Environment, IEEE INFOCOM, volume 3, pages 1548-1557, 2001.

[13] Gaddallah, Y. and Kunz, T., Energy consumption in ad-hoc routing protocols: Comparing DSR, AODV and TORA, Proceedings of the 1st International Conference on Ad-Hoc Networks and Wireless, Toronto, Canada, pages 161-176, September 2002. 
[14] Haas, Z.J. and Pearlman, M.R., The Zone Routing Protocol (ZRP) for Ad Hoc Networks, Internet Draft, available on line [accessed on March 10, 2004] http://www.ietf.org/proceedings/99jul/I-D/draft- ietf-manet-zone-zrp-02.txt

[15] Johnson, D., Maltz, D., and Broch, J., DSR: The Dynamic Source Protocol for Multi-hop Wireless Ad hoc Networks, in Ad hoc Network edited by Charles Perkins, Addison-Wesley, pages 139-172, 2001.

[16] Jones, C.E., Sivalingam, K. M., Agrawal, P., Chen, J., A survey of energy Efficient Network Protocols for Wireless Networks, ACM Journal on Wireless Networks, pages 343-358, 2001.

[17] NS2 manual, available on line [accessed on March 10, 2004] http://www.isi.edu/nsnam/ns/

[18] Kim, D. and Garcia-Luna-Aceves, J.J., Performance Analysis Of Power-Aware Route Selection Protocols in Mobile Ad hoc Networks, IEEE Networks 2002: joint conference ICWLHN 2002 and ICN 2002, Atlanta, Georgia, August 26$29,2002$.

[19] Ko, Y. and Vaidya, N., Location-aided Routing (LAR) in Mobile Ad hoc Networks, Proceedings of the Fourth Annual ACM/IEEEE International Conference on Mobile Computing and Networking, pages 66-75, 1998.

[20] Kravets, R., Krishnan, P., Power management techniques for Mobile communication, Proceedings of the $4^{\text {th }}$ annual ACM/IEEE international conference on Mobile computing and networking, pages 157-168, 1998. 
[21] Li, Q., Aslam, J., and Rus, D., Online Power-aware routing in Wireless Ad-hoc Networks, Proceedings of the ACM Mobile Computing and Network Conf., Rome, Italy, pages 97-107, July 2001.

[22] Macker, J. and Corson, S., Mobile Ad hoc Networking (MANET): Routing Protocol Performance Issues and Evaluation Considerations, RFC2501, available on line [accessed on March 10, 2004] www.ietf.org/rfc/rfc2501.txt

[23] Maleki, M., Dantu, K., and Pedram, M., Power-aware Source Routing Protocol for Mobile Ad Hoc Networks, Proceedings of the 2002 international symposium on Low power electronics and design, pages 72-75, 2002.

[24] Maltz, D.A., On-Demand Routing in Multi-hop Wireless Mobile Ad Hoc Networks, Ph.D. thesis, May 2001.

[25] Marina, M.K. and Das, S.R., On-Demand Multipath Distance Vector Routing in Ad Hoc Networks, Proceedings of the International Conference for Network Protocols, pages 14-23, 2001.

[26] Michail, A. and Ephremides, A., Energy-efficient routing for ConnectionOriented Traffic in wireless Ad-hoc Networks, Mobile Networks and Applications, Volume 8, Issue 5, pages 517-533, 2003.

[27] Murthy, S. and Garcia-Luna-Aceves, J. J., An Efficient Routing Protocol for Wireless Networks, ACM Mobile Networks and Applications Journal 
(MANET), Special Issue on Routing in Mobile Communication Networks, Vol.1, No. 2, pages 183-197, October 1996.

[28] Nasipuri, A. and Das, S.R., On-Demand Multipath routing for Mobile Ad hoc Networks, Proceedings of the International Conference for Network Protocols (ICNP), pages 14-23, November 2001.

[29] Perkins, C.E. and Royer, E. M., Multicast Operation of the Ad-hoc On-demand Distance Vector Routing Protocol, Proceedings of MobiCom '99, pages 207218, Seattle, WA, August 1999.

[30] Perkins, C.E. and Royer, E.M., Ad-hoc On-Demand Distance Vector Routing, Proceedings of the 2nd IEEE Workshop on Mobile Computing Systems and Applications (WMCSA'99), New Orleans, LA, USA, pages 90100, February 1999.

[31] Perkins, C.E. and Bhagwat, P., Highly Dynamic Destination-Sequenced Distance-Vector Routing (DSDV) for Mobile Computers, ACM/SIGCOMM'94 Conference on communications Architectures, Protocols and Applications, pages 234-244, October 1994.

[32] Qin, L. and Kunz, T., Pro-active route maintenance in DSR, ACM Mobile Computing and Communications Review, Vol. 6, No. 3, pages 79-89, July 2002. 
[33] Ramanathan, R. and Regina, R.-H., Topology control of multihop wireless networks using transmit power adjustment, IEEE Proceedings of Infocom, volume 2, pages 404-413, 2000.

[34] Rappaport, T. S., Wireless Communications: Principles and Practice $\left(2^{\text {nd }}\right.$ Edition), Chapter 9, pages 439-482, Prentice Hall, ISBN: 0130422320, 2002.

[35] Rodoplu, V. and Meng, T., Minimum Energy Mobile Wireless Networks, IEEE Journal of Selected Areas in Communications, vol.17, no.8, pages 13331344, 1999.

[36] Royer, E.M. and Toh, C.-K., A Review of Current Routing Protocols for AdHoc Mobile Wireless Networks, IEEE Personal Communications Magazine, pages 46-55, April 1999.

[37] Singh, S., Woo, M., and Raghavendra, C.S., Power-Aware Routing in Mobile Ad Hoc Networks, Proceedings of the $4^{\text {th }}$ annual ACM/IEEE international conference on Mobile computing and networking, Dallas, pages 181-190, 1998.

[38] Stojmenovic, I. and Lin, X., Power-aware localized routing in wireless networks, IEEE Trans. Parallel Distribution Systems, Vol. 12, No. 11, pages 1122-1133, October 2001.

[39] Subbarao, M.W., Performance of Routing Protocols for Mobile Ad hoc Networks, available on line [accessed on March 10, 2004] http://w3.antd.nist.gov/wctg/manet/docs/perf_routing_protocols.pdf 
[40] Toh, C.K., Maximum Battery Life Routing to support Ubiquitous Mobile Computing in Wireless Ad hoc Networks, IEEE communication Magazine, volume 39, pages 138-147, June 2001.

[41] Wan, P.J., Calinescu, G., Li, X., and Frieder, O., Minimum-Energy Broadcast Routing in Static Ad Hoc Wireless Networks, Wireless Networks, Volume 8, Issue 6, pages 607-617, 2002.

[42] Wu, J., An Extended Dynamic Source Routing scheme in Ad hoc Wireless Networks, Proceedings of the 35th Hawaii International Conference on System Sciences (HICSS02), pages 296-303, 2002.

[43] Wu, J., Dai, F., Gao, M., and Stojmenovic, I., On calculation Power-Aware Connected Dominating Sets for Efficient Routing in Ad Hoc Wireless Networks, Journal of Communications and Networks, Vol. 5, No. 2, pages169178, March 2002.

[44] Wu, K. and Harms, J., On-Demand Multipath Routing for Mobile Ad Hoc Networks, Journal of Communications and Networks Special Issue on Innovations in Ad Hoc Mobile Pervasive Network, Vol.4, Number 1, page 4858, March 2002.

[45] Xu, Y., Heidemann, J. and Estrin, D., Adaptive energy-conserving routing for multihop ad hoc networks, Technical Report TR-2000-527, 2000. 


\section{Appendixes}

\section{Energy efficient routing protocol simulation using NS2}

Simulation environment:

Operating System: Windows

NS2 version: ns-allinone-2.1b9a

NS2: download from URL: http:/www.isi.edu/nsnam/ns/index.html

\section{NS2 mobile node network components}

Each component is described in [17]. The mobile node network stack consists of several levels: link layer (LL), ARP module connected to LL, interface priority queue (Ifq), Mac layer (MAC), a network interface (netIF). All the levels are connected to the primary channel. These network components are implemented in Otcl. Figure 21 taken from [17] is schematic of a DSR mobile node.

\section{LL (Link Layer):}

The LL in mobile node network has an ARP module connected to it which resolves all IP to hardware (Mac) address conversions. Normally for all outgoing (into the channel) packets, the packets are handed down to the LL by the Routing Agent. The LL hands down packets to the interface queue. For all incoming packets (out of the channel), the Mac layer hands up packets to the LL, which is then handed off at the 
node_entry_ point. The class LL is implemented in ns-allinone-2.1b9a/ns-2.1b9a /11. $\{\mathrm{cc}, \mathrm{h}\}$.

ARP :

The Address Resolution Protocol (implemented in BSD style) module receives queries from Link layer. If ARP has the hardware address for destination, it writes it into the Mac header of the packet. Otherwise it broadcasts an ARP query, and caches the packet temporarily. For each unknown destination hardware address, there is a buffer for a single packet. Incase additional packet to the same destination is sent to ARP, the earlier buffered packet is dropped. Once the hardware address of a packet's next hop is known, the packet is inserted into the interface queue. The class ARPTable is implemented in ns-allinone-2.1b9a/ns-2.1b9a /arp. $\{c c, h\}$ and nsallinone-2.1b9a/ns-2.1b9a /tcl/lib/ns-mobilenode.tcl.

\section{Ifq (Interface Queue):}

The class PriQueue is implemented as a priority queue that gives priority to routing protocol packets, inserting them at the head of the queue. It supports running a filter over all packets in the queue and removes those with a specified destination address. The interface queue is implemented in ns-allinone-2.1b9a/ns-2.1b9a /priqueue. $\{\mathrm{cc}, \mathrm{h}\}$.

\section{Mac Layer (Media Access Control):}

The IEEE 802.11 distributed coordination function (DCF) Mac protocol has been implemented by CMU. It uses a RTS/CTS/DATA/ACK pattern for all unicast 
packets and simply sends out DATA for all broadcast packets. The implementation uses both physical and virtual carrier sense. The class Mac802_11 is implemented in ns-allinone-2.1b9a/ns-2.1b9a/mac-802_11.\{cc,h\}.

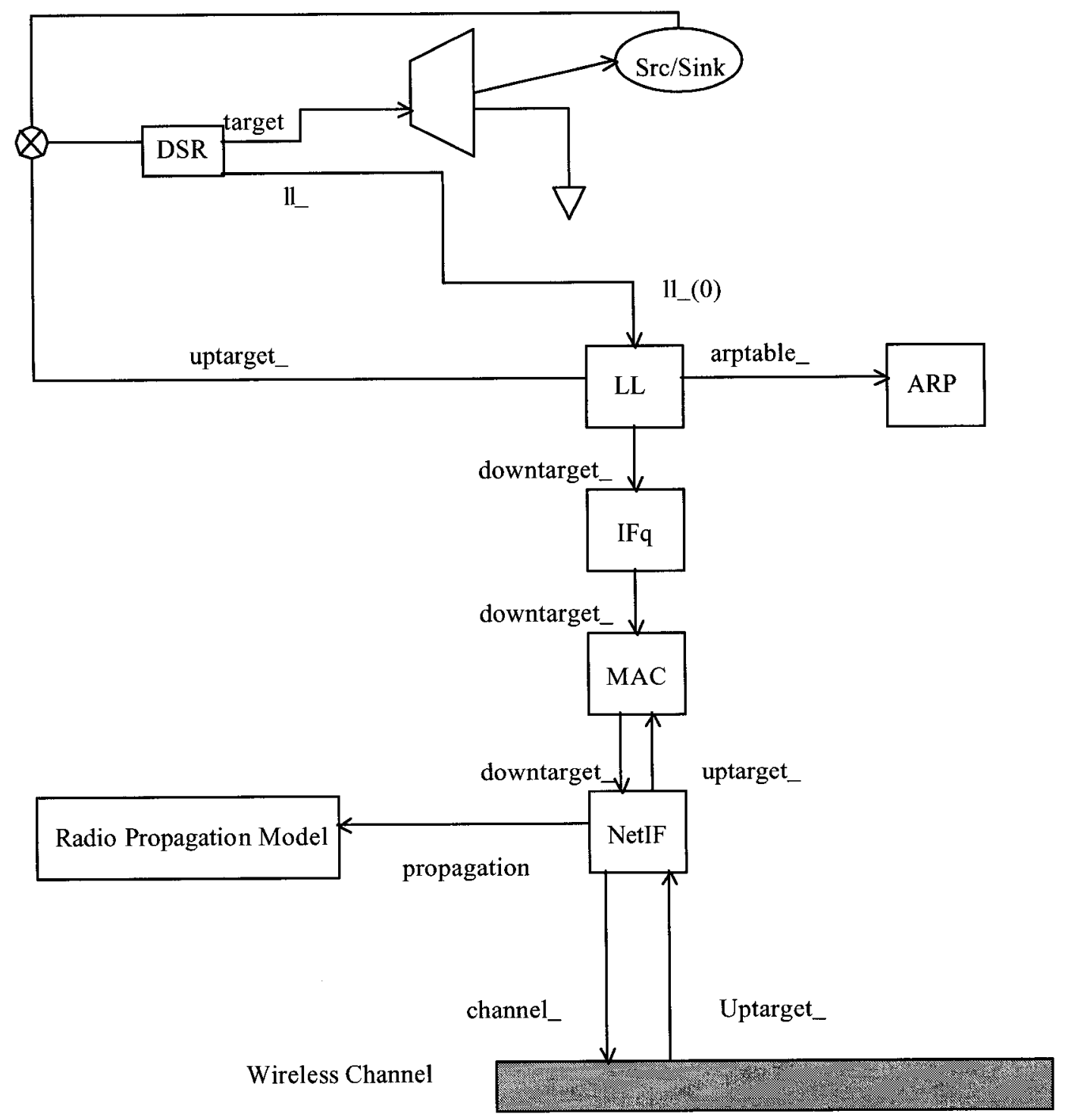

Figure 21. Mobile node network components in DSR 


\section{Tap Agents:}

Agents that subclass themselves as class Tap defined in mac.h can register themselves with the Mac object using method installTap(). If the particular Mac protocol permits it, the tap will promiscuously be given all packets received by the Mac layer, before address filtering is done. The class Tapmplementation is in nsallinone- $2.1 \mathrm{~b} 9 \mathrm{a} / \mathrm{ns}-2.1 \mathrm{~b} 9 \mathrm{a} / \mathrm{mac} .\{\mathrm{cc}, \mathrm{h}\}$.

\section{NetIF ( Network Interfaces ):}

The Network Interphase layer serves as a hardware interface that is used by mobile node to access the channel. The wireless shared media interface is implemented as class Phy/WirelessPhy. This interface subject to collisions and the radio propagation model receives packets transmitted by other node interfaces to the channel. The interface stamps each transmitted packet with the meta-data related to the transmitting interface like the transmission power, wavelength etc. This meta-data in pkt header is used by the propagation model in receiving network interface to determine if the packet has minimum power to be received and/or captured and/or detected (carrier sense) by the receiving node. The model approximates the DSSS radio interface (Lucent WaveLan direct-sequence spread-spectrum). The class is implemented in ns-allinone-2.1b9a/ns-2.1b9a/wireless-phy. $\{\mathrm{cc}, \mathrm{h}\}$.

\section{Radio Propagation Model:}


It uses Friss-space attenuation $\left(1 / \mathrm{r}^{2}\right)$ at near distances and an approximation to two ray Ground $\left(1 / \mathrm{r}^{4}\right)$ at far distances. The approximation assumes specular reflection off a flat ground plane. The class is implemented in ns-allinone-2.1b9a/ns-2.1b9a /tworayground. $\{\mathrm{cc}, \mathrm{h}\}$.

\section{Antenna:}

An omni-directional antenna having unity gain is used by mobile node. The class is implemented in ns-allinone-2.1b9a/ns-2.1b9a/antenna. $\{c c, h\}$.

\section{Protocol implement}

We designed EPDSR based on DSR using NS2. We modified some of the code files in dsragent.cc, dsragent.h, energy-model.cc. The following is the description of the modifications that have been made:

- In energy-model.cc, change the energy model using the function (2) in Chapter 4.3.5 and using the parameters in Table 2.

- In srpacket.h, add double variable cost and Time variable time. Cost represents the cost on the route and times represents the time when the packet arrives.

- In dsragent.cc, monitor the intermediate node that received srpacket, compare the cost and decides whether forward it or discard it.

- In dsragent.cc, monitor the destination node that received srpacket, compare the cost and decides the choice of the route.

- In dsragent.cc, monitor the cost change of each node along the route.

If the cost change of each node on the route exceeds the limitation, delete this 
route.

- In dsragent.cc, deal with DSRagent::getRouteForPacket. When there is no route to the destination, issues two request packets.

\section{Mobile Node Movement Scenario Files Creation}

The node movement generator is available under ns-allinone-2.1b9a/ns-2.1b9a /indep-utils/cmu-scen-gen/setdest directory and consists of setdest $\{. \mathrm{cc}, \mathrm{h}\}$ and Makefile. In order to compile the revised setdest.cc do the following:

1) Go to ns directory and run "configure". This creates a makefile for setdest.

2) Go to indep-utils/cmu-scen-gen/setdest. Run "make", which first creates a standalone object file for $\sim$ ns/rng.cc and then creates the executable setdest.

3) Run setdest with arguments as following:

./setdest [-n num_of_nodes] [-p pausetime] [-s maxspeed] [-t simtime] $[-\mathrm{x} \operatorname{maxx}][-\mathrm{y} \operatorname{maxy}]>[$ output-file $]$

\section{Traffic Pattern Scenario Files Creation}

Random traffic connections of TCP and CBR can be set up between mobile nodes using a traffic-scenario generator script. This traffic generator script is available under ns-allinone-2.1b9a/ns-2.1b9a/indep-utils/cmu-scen-gen and is called cbrgen.tcl. It can be used to create CBR and TCP traffics connections between wireless mobile nodes. In order to create a traffic-connection file, we need to define the type of traffic connection (CBR or TCP), the number of nodes and maximum number of connections to be set up between them, a random seed and in case of 
CBR connections, a rate whose inverse value is used to compute the interval time between the CBR packets. So the command line looks like the following:

ns cbrgen.tcl [-type cbr|tcp] [-nn nodes] [-seed seed] [-mc connections] [-rate rate] $>$ [out-put file]

During the creation of the traffic pattern, we use the CBR pattern type, and change the default packet size 512 bytes to different size from 1024 bytes to 64 bytes. 